\title{
Association of Crocus sativus with Cognitive Dysfunctions and Alzheimer's Disease: A Systematic Review
}

\author{
Marjan Talebi $^{1}{ }^{(\mathbb{D}}$, Mohsen Talebi ${ }^{2}$ (D), Saeed Samarghandian ${ }^{3, *}$ \\ 1 Department of Pharmacognosy and Pharmaceutical Biotechnology, School of Pharmacy, Shahid Beheshti University of \\ Medical Sciences, Tehran, Iran; talebi.m@ sbmu.ir; \\ 2 Food Safety Net Services, San Antonio, Texas 78216, USA; mohsen.talebi@fsns.com; \\ 3 Healthy Ageing Research Center, Neyshabur University of Medical Sciences, Neyshabur, Iran; \\ samarghandians1@nums.ac.ir; \\ * Correspondence: samarghandains1@nums.ac.ir;
}

Scopus Author ID 6507632790

Received: 8.05.2020; Revised: 10.06.2020; Accepted: 13.06.2020; Published: 17.06.2020

\begin{abstract}
Alzheimer's disease (AD) is known as a principle basis of cognitive impairment in the elderly population. Current available therapeutic approaches are not applicable enough for the reason of intolerable side effects, low patients' compliance due to the short half-life, and biopharmaceutical limitations. Therefore discovery alternative and multi-targeted therapeutic approaches are sought. Crocus sativus is a distinguished medicinal plant with a wide range of biological and pharmacological belongings, exclusively antioxidant and anti-inflammatory possessions. Following PRISMA guidelines, a systematic review was conducted by search in Electronic databases comprising Scopus, PubMed, Web of Science, and Embase using the keywords cognitive OR cognition OR memory OR nootropic OR Alzheimer OR amnesia OR dementia AND "Crocus sativus" OR saffron OR crocin OR crocetin OR safranal until 21 st February 2020. The results display that saffron and its major constituents are capable of having remarkable properties on memory and cognitive deficiency. Cell signaling pathways, antioxidative stress experiments, modulation in inflammatory and proinflammatory mediators, tauopathy, clearance of amyloid-beta aggregation, and histopathological alterations are indicated. Cognition tests and scores allied with memory loss are specified. The administration of saffron in experimental models seems to be an encouraging attitude in $\mathrm{AD}$ even though it is recommended that further studies for the valuation of pharmacokinetic properties and bioavailability enhancing carriers of these phytochemicals must be directed.
\end{abstract}

Keywords: Alzheimer's disease; amnesia; cognitive disorders; crocetin; crocin; dementia; memory impairment; nootropic; saffron; safranal.

(C) 2020 by the authors. This article is an open-access article distributed under the terms and conditions of the Creative Commons Attribution (CC BY) license (https://creativecommons.org/licenses/by/4.0/).

\section{Introduction}

Neurodegenerative diseases, described by irreversible neuron loss and gliosis (nonspecific changes of glial cells), including disorders such as Parkinson's disease (PD), frontotemporal degeneration (FTD) and Alzheimer's disease (AD) [1]. AD is postulated through the process of getting old and the most prevalent reason for dementia in aged people. AD shows, unlike clinical manifestations; however, cognitive dysfunctions and memory loss are the main hallmarks that can lead to subsequent incapability and death [2]. AD recruits with diminishing in memory, and according to its progression, AD can be classified into three phases: a. the preclinical phase, there is not any modification in cognitive ability, $b$. the mild cognitive impairment (MCI) phase, and c. dementia phase [3]. The global annual expenses of 
dementia were assessed (US) \$818 billion in 2015. Besides, AD may inflict passionate stresses on patients and their relatives. It has been appraised that 115.4 million individuals will suffer from AD until the year $2050[3,4]$.

\subsection{Pathogenesis of Alzheimer's Disease.}

The hippocampus is an anatomical construction in the brain medial temporal lobe that is imperative for learning and memory. It consists of four subregions, which consist of the dentate gyrus (DG), hippocampus proper (CA1, CA2, and CA3), subicular complex, and entorhinal cortex (EC). A short-term high-frequency train of inducements to major synaptic pathways raises the amplitude of the excitatory postsynaptic potentials in the target hippocampal neurons. This enablement is entitled long-term potentiation (LTP), which is substantial in the duty of memory deficiencies. Abundant convoluted mechanisms anticipated in the pathogenesis of $\mathrm{AD}$ [5]. There is a low chance (5-10\%) for the incidence of genetic history. AD is associated with mutations in three different genes: presenilin-1 (PS1), presenilin2 (PS2), and amyloid- $\beta$ precursor protein (APP- $\beta$ ) [6-10]. Aggregation of $\beta$-amyloid protein $(\mathrm{A} \beta)$ in neuritic plaques (NPs) and hyper-phosphorylation of tau $(\tau)$ protein (tauopathy) in neurofibrillary tangles (NFTs) are the most momentous pathophysiological changes [11-14]. The formation of $A \beta$ deposits leads to an immune response with the contribution of astrocytes and microglia [5,11]. Reactive microglia can produce free radicals, which can be fatal for neurons. Activation of some T-cells has been observed in brain parenchyma of AD sufferings. Such cells release inflammatory mediators, including; interleukin (IL)-1, IL6, $\gamma$-interferon, and tumor necrosis factor-alpha $(\mathrm{TNF}-\alpha)$.It has approved that usage of non-steroidal antiinflammatory drugs (NSAIDs) modulates the inflammatory factors responsible in AD through inhibition of cyclo-oxygenase (COX). Evidence-based studies are endorsed effects of oxidative stress on augmentation of formation and accumulation $A \beta$ and hyper-phosphorylation of tau. It was observed that alteration in levels of catalase (CAT), superoxide dismutase (SOD), glutathione peroxidase (GSH-Px), and total antioxidant status (TAS) in erythrocyte and the plasma could be considered as a marker for pathological distinctions in the brain of patients with AD [15]. Environmental factors and apolipoprotein-E are claimed as other probable causes of $\mathrm{AD}[16,17]$.

\subsection{Therapeutic Strategies in Alzheimer's Disease.}

As a reason for the intervention of multiple factors in the initiation of $\mathrm{AD}$, a multitarget-directed ligand strategy will be an effective therapeutic approach [18]. There are two groups of FDA-approved medications for the management of AD symptoms. These drugs act through two mechanistic pathways; Non-competitive N-methyl-D-aspartate (NMDA) receptor antagonists (memantine) and cholinesterase inhibitors (ChEIs) (donepezil, galantamine, and rivastigmine) $[19,20]$. These chemicals, synthetic therapeutic agents, have some side effects, short half-life, and fluctuated bioavailability, which leads to narrow indications [18]. There is a growing demand for the usage of phytochemicals, nutraceuticals, and phytochemical components for the control and management of numerous diseases such as AD. Phosphatidylserine, Ginko, huperzine, and vinpocetine are obtainable natural-based products in the market, which can ameliorate mental functions in patients who have dementia. By considering the tendency to discover novel drugs and the opulent therapeutic potential of 
phytochemicals, investigation of the anti-AD or memory-enhancing effects of those would be well-intentioned [16,21-24].

\subsection{Phytochemical Properties of Saffron.}

Saffron is botanically named as Crocus sativus L. (Iridaceae) has three major components; crocins, (the source of coloring pigment), the glycoside picrocrocin (a precursor of safranal and contributed its explicit flavor), and safranal (the deglycosylated form of picrocrocin and the major organoleptic principle of the stigmas) $[25,26]$. Crocin, crocetin are carotenoids of saffron, and safranal is a monoterpene aldehyde [27,28]. Carotenoids are bioactive components with great antioxidant potential [29]. The high-performance liquid chromatography (HPLC), ionization-mass spectrometry (APCI-MS), (Fourier transform nearinfrared) FT-NIR, and ultraviolet-visible (UV-Vis) spectrometry analytical procedures are chiefly applied for qualitative and quantitative analysis of saffron components [30,31].

\subsection{Biological and Pharmaceutical Aspects of Saffron.}

Numerous pharmacological effects have been endorsed to saffron and its components including; anticancer [32,33], neuroprotective (anti-Alzheimer's disease, anti-Parkinson, anticonvulsant, antidepressant, anxiolytic, and anti-schizophrenia) [34-37], anti-ischemic (heart, muscular, kidney, and brain ischemia, ) [38], antioxidant [39], antinociceptive, antitussive, hypolipidemic, anti-diabetic [40], antidote (against snake venom, acrylamide, etc.), anti-obesity and anorectic, Aphrodisiac, nephroprotective [41], anti-hypertensive, antigenotoxic, cardioprotective [42], antimicrobial, hepatoprotective, gastroprotective [43] and anti-inflammatory effects [44-46]. Saffron is wildly used in food flavoring and coloring, antipruritic and emollient, cosmetics, textile dye, perfume, and pharmaceutical industry $[47,48]$.

\section{Study Design}

Herein we conducted the systematic review coincident to the established PRISMA guidelines [49]. A literature search was targeted on the electronic databases of Scopus, PubMed, Embase, and Web of Science. Other databases were searched to avoid missing related articles. There was no time limitation for the searched articles. The search was accomplished by using the following search strings in the title/abstract/keywords: cognitive OR cognition OR memory OR nootropic OR Alzheimer OR amnesia OR dementia AND "Crocus sativus" OR saffron OR crocin OR crocetin OR safranal. Reclaimed articles were imported to EndNoteX6 reference management software. All articles were separately screened for, duplicity, and eligibility by authors individually.

\subsection{Inclusion Criteria.}

Articles were included with the following properties; I) original research articles, II) articles published in English, III) use of any form of Crocus sativus and its major constituents (crocin, safranal, and crocetin), IV) researches with sufficient outcomes associated with objectives of the study and V) published and in press articles before 21st February 2020. 


\subsection{Exclusion Criteria.}

Articles were excluded with the following properties; I) the study did not evaluate AD, cognitive disorders, and any related conditions of memory loss, II) no related experimental assays were used III) Crocus sativus or its constituents were used as a positive control or as bio-enhancers IV) only abstract was available V) the study directly claimed that crocin was originated from gardenia.

\subsection{Data Extraction.}

In total, the search yielded 451 articles of those 92 met inclusion criteria (Figure 1). In total, 60 studies were based on animal-based experiments, 29 studies were based on cellular or in vitro studies, and 8 studies were based on human clinical trials. Some of the selected articles had in vitro and in vivo studies both. In vivo studies containing nonspecific experiments were not categorized as a separate result. Studies evaluating AD, other cognitive incapacities due to neuronal disturbances, and examining healthy cognition performance were included.

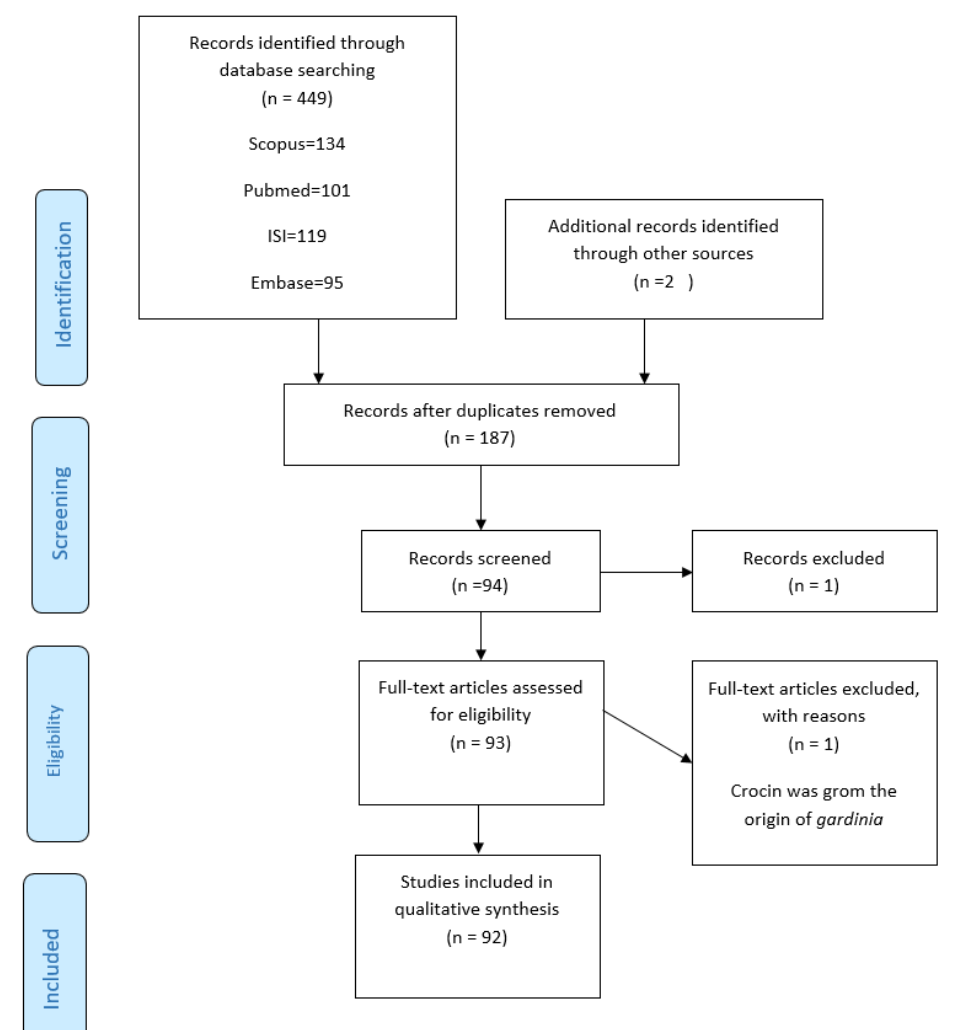

Figure 1. Study Selection Diagram.

\section{Mechanisms Involved in Memory Enhancing and anti-Alzheimer's Disease Potential of Crocus sativus}

This is the first systematic review for evaluation of the association between consumption of saffron and its therapeutic approach in Alzheimer management and cognitive dysfunctions. Characterized extracted data of in vivo, in vitro, and clinical studies are collected in (Table 1-3), respectively.

In animal-based studies, some cognition measuring experiments were performed, and associated results show memory enhancement. Open Field Test (OFT) has indicated an increase in a. the number of square crosses, b. the number of rearings, c. time spent in the center 
and number of center entries.Y-Maze Test has demonstrated an elevation in a. spontaneous alterations (\%) and b. the number of arm entries. Passive Avoidance Test (PAT) has shown a. increase in Step-Through Latency (STL) and b. the decrease in Initial Latency (IL). Morris Water Maze (MWM) is used for examination of spatial learning and memory, which has indicated a. attenuation in Escape Latency (EL), b. increase in Time Spent in Target Quadrant (TSTQ), and c. increase in swimming speed. Radical 8-Arm Maze (RAM) has demonstrated a reduction in working memory errors and reference memory errors. Novel Object Recognition Test (NORT) is shown elevation in a. Discrimination Index (DI) and b. Exploration time of the novel object. Other memories assessing behavioral experiments such as; Novel Object Location Test (NOLT) Elevated Plus Maze (EPM), Barnes Maze, T-Maze, and Sucrose Performance Test were also used (142) [50-53]. Improvement of the cognitive dysfunctions or memory impairments through pretreatment with saffron and its constituents is demonstrated apparently in preclinical studies [45].

Clinical trials, in vivo and in vitro, showed that $\mathrm{A} \beta$ acts a main function in the pathogenesis of $\mathrm{AD}$. $\mathrm{A} \beta$ accumulation is responsible for senile plaques formation, activation of oxidative damage, neuronal inflammation, apoptotic death, and cognitive impairments. Mitigating the ROS formation via the consumption of saffron, safranal, crocin, and crocetin is one of the significant mechanisms in the AD therapeutic approach. Increase SOD, CAT, GSH, oxidized glutathione (GSSG), and TAC and alleviation of Malondialdehyde (MDA), nitric oxide (NO), Lactate dehydrogenase (LDH), carbonyl proteins and DNA damage are desired (143) [54-56]. Obtaining free radical scavenging capacity is probable through 2,2,1-diphenyl1-picrylhydrazyl (DPPH), Thiobarbituric acid reactive substances (TBARS) and ferric reducing antioxidant power (FRAP), as well. Attenuation of the $\beta$-cleavage of APP may lead to protection combat $\mathrm{AD}(144,145)$. The balance of pro- and anti-apoptotic Bcl-2 family proteins (e.g., Bcl-2 and Bcl-w versus Bad, Bim, and Bax) plays a pivotal function in apoptosis neuronal cells. Thus expression levels of these proteins are seemingly changed in the vulnerable neurons in $\mathrm{AD}$ [57].

Exposure to $A \beta$ peptide initiates the activation of astrocytes and microglia leads to proinflammatory chemokines and cytokines such as IL-8, IL-6, IL-1 $\beta$, and TNF- $\alpha$ [58]. Elevation in IL-1 $\beta$ level is accompanied by over activation of c-jun-N-terminal kinases (JNK), caspase-3, and mitogen-activated protein kinase (MAPK),; all of these contributed to apoptosis and synapse loss. IL-1 $\beta$ also attenuates the release of acetylcholine in synaptic space, which contributes to further cognitive impairments in AD. The release of chemokines can induce the migration of monocytes from the peripheral circulation into the brain and initiates inflammatory responses (146), whereas pretreatment with saffron attenuated its activations and regulation of p38 MAPK, and AKT pathways [59]. Lipocalin-2 (LCN2) is a principle molecule that acts in cell viability, inflammation, and many other biological reactions. LCN2 manipulations may be a potential therapeutic target in CNS inflammatory conditions for instance, $\mathrm{AD}$ [60]. Activation of the $78-\mathrm{kDa}$ glucose-regulated protein (GRP78) of the endoplasmic reticulum may participate in phosphatidylinositol 3-kinase (PI3K)/AKT pathway of management the neurological disorders [61]. Transcription factor nuclear factor-kappa B $(\mathrm{NF}-\kappa \mathrm{B})$ is a crucial regulator of innate immunity. NF- $\kappa \mathrm{B}$ may regulate AD pathophysiologically caused by apolipoprotein-E, metabolic syndrome, APP, ROS production, and environmental factors [62]. Neurotrophin BDNF plays the role of regulating diversified neuronal structure and function in the development of the adult CNS and has known as one of the most significant signaling molecules for the development of the nervous system. It has been 
reported that BDNF plays a critical role in neuronal survival, synaptic plasticity, and memory [63]. Cyclic AMP (cAMP)-responsive element-binding protein (CREB) is a nuclear transcription factor that contributed to $\mathrm{AD}[64]$.

$\mathrm{A} \beta$ consists of 36-43 amino acids and is a natural product of sequential cleavage of an integral membrane protein, the amyloid precursor protein (APP). APP can undergo cleavage in one of two non-amyloidogenic and amyloidogenic pathways. In the first pathway, cleavage by the enzyme $\alpha$-secretase prevents A $\beta$ formation. It leads to the production of the sAPP $\alpha$ fragment that has some beneficial properties such as promoting neuronal growth and neuroprotective effects. However, the sequential cleavage by $\gamma$-secretase(containing presenilin 1 at its catalytic core) and $\beta$-secretase, a beta-site amyloid precursor protein-cleaving enzyme 1 (BACE-1) lead to the formation of $A \beta$. Then $A \beta$ releases into the extracellular spaces. Mediation APP and BACE-1 are considered as effective pathways in the mitigation of memory impairment by saffron [65]. Inhibition of activator and signal transducer of transcription 3 (STAT3) is a molecular mechanistic target combat AD and cognitive dysfunctions [66].

Consequently, JAK/STAT3 cascade and Glial Fibrillary Acidic Protein (GFAP) run as a dominant controller in astrocyte goings-on [67]. Postsynaptic density protein 93 (PSD-93) and PSD-95 are significant scaffolding proteins in the regulation of A $\beta$-persuaded synaptic dysfunctions [68]. Neutralization caspases activities or expression are considered as therapeutic targets combat neurodegenerative disorders like AD [69]. Peripheral blood mononuclear cells are considered as $\mathrm{A} \beta$ plaque suppressors through modulating $\mathrm{BBB}$ penetration by chemokines such as CXCL-10 [70]. CHOP gene (C/EBP-homologous protein) is a pro-apoptotic transcriptional factor that may cause $\mathrm{AD}$ in vivo, then deactivating this gene will be an option for the discovery of novel drugs with anti-AD properties [71]. It has been demonstrated by augmentation in levels of corticosterone risk of $\mathrm{AD}$ induction increased through $\mathrm{A} \beta$ plaque formation [72]. Matrix metalloproteinases (MMPs) are responsible for neuroinflammation in the pathogenesis of AD, MMP-2, MMP-3, and MMP-9 are the most important ones that are correlated with $A \beta$ plaque formation [73]. An extracellular signal-regulated kinase (ERK) plays a functional role in restoring neuronal damages [74]. Insulin-like growth factor 1 (IGF-1) may play a pivotal role in the progression and pathogenesis of $\mathrm{AD}$ [75]. The p53 protein has appeared to intermediate programmed cell death in neurons of AD by regulating levels of $\mathrm{Bcl} 2$ family proteins [76].

TAU hyperphosphorylation can alter various synaptic proteins via inhibition of PP2A and activation of GSK-3 $\beta$. Therefore synaptic proteins and their levels of expression may cause an anti-inflammatory effect in the hippocampus of AD [77]. Mammalian target of rapamycin (mTOR) may correlate in $\mathrm{A} \beta$ plaque formation and tauopathy induced neurodegeneration [78]. Machine learning and pattern recognition techniques are appropriate for diagnosing AD and MCI from individual MRI scans. Another application of such methods is to predict clinical scores from individual scans. Relevance vector regression (RVR), the Mini-Mental State Examination (MMSE), Dementia Rating Scale (DRS), and Auditory Verbal Learning Test (AVLT) are the basic methods. Prediction and actual clinical scores are evaluated by Alzheimer's Disease Assessment Scale-Cognitive (ADAS-cog), MMSE, and DRS tests. This result underscores their utility for screening and tracking disease. In clinical practice, we visualize the usage of RVR to help practitioners diagnose and predict clinical outcomes. Individual MMSE items, especially those with some type of episodic memory referent, were the best predictors of incident cases of AD. Moreover, MMSE items displayed fluctuation, especially in the incidence of $\mathrm{AD}$ (147). 
Table 1. Results of in vivo studies.

\begin{tabular}{|c|c|c|c|c|c|}
\hline $\begin{array}{l}\text { Animal/ } \\
\text { Sample size } \\
\text { (No. of } \\
\text { groups) }\end{array}$ & $\begin{array}{l}\text { Model of } \\
\text { Study }\end{array}$ & $\begin{array}{l}\text { Targeted compound } \\
\text { /Duration/ } \\
\text { Dosage/ } \\
\text { Route of } \\
\text { Administration } \\
\end{array}$ & $\begin{array}{l}\text { Cognition } \\
\text { Test }\end{array}$ & Major Outcomes & Ref. \\
\hline Rat/ 42,6 & malathion & $\begin{array}{l}\text { Crocin/14 days/10, } \\
20 \text { and } 40 \mathrm{mg} / \mathrm{kg} / \text { i.p. }\end{array}$ & MWM & $\begin{array}{l}\downarrow \text { EL, } \uparrow \text { TSTQ, } \uparrow \text { swimming } \\
\text { distance, AChE activity=>NSD, } \\
\downarrow \text { MDA, } \uparrow \text { GSH }, \downarrow \text { TNF- } \alpha, \downarrow \text { IL-6, } \downarrow \\
\text { PSD93 protein level, } \downarrow \text { TAU } \\
\text { protein level at Thr205 and } \\
\text { Ser404, phosphorylated and total } \\
\text { GSK-3 } \beta=>\text { NSD, } \downarrow \text { PP2A at the } \\
\text { pY307-PP2AC protein level, } \\
\downarrow \text { TAU mRNA expression, } \uparrow \text { PP2A } \\
\text { mRNA level, } \downarrow \text { Bax/Bcl2 ratio, } \\
\text { caspases }(3,8 \text { and } 9)=>\text { inhibited }\end{array}$ & [77] \\
\hline Mouse/ 56, 3 & C57BL/6 J & $\begin{array}{l}\text { Trans-crocin-4 } \\
\text { (TC4)/50, } 150 \\
\text { mg/kg/ i.p. }\end{array}$ & N.M. & 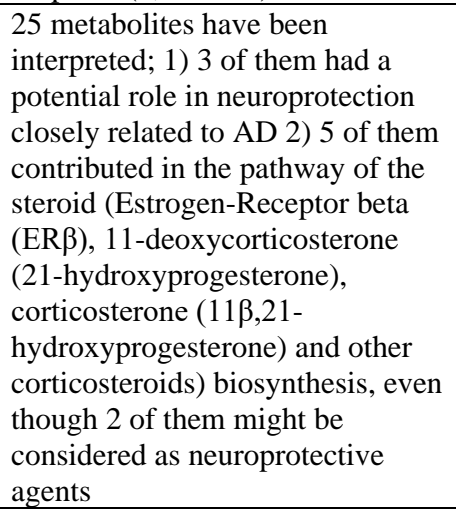 & [79] \\
\hline Rat/ 46, 4 & Lipocalin-2 & $\begin{array}{l}\text { Saffron in a } \\
\text { combination/ } 28 \\
\text { days/ } 16.5,33 \mathrm{mg} / \mathrm{kg} \\
\text { p.o. }\end{array}$ & MWM & $\begin{array}{l}\downarrow \text { Neurological score after } 2 \mathrm{~h}, \\
\downarrow \text { Neurological score after } 24 \mathrm{~h}, \\
\downarrow \text { Brain Damage after Surgery, } \\
\downarrow \text { number and size of infarcts, } \\
\text { nerve cell degeneration, and } \\
\text { Necrosis in the infarcts=> Were } \\
\text { not observed, necrotic } \\
\text { substances=>-were absorbed, } \downarrow \text { EL, } \\
\uparrow T S T Q, \uparrow p l a t f o r m \text { crossings, } \downarrow \mathrm{IL}- \\
1 \alpha, \downarrow I L-12, \downarrow \text { CXCL10, } \downarrow \text { LCN2 } \\
\text { secretion, } \downarrow \text { p-STAT3 and p-JAK2 } \\
\text { staining in astrocytes, } \downarrow \text { OGD- } \\
\text { Induced Injury in Astrocytes }\end{array}$ & [80] \\
\hline Mouse/ 60 & $\mathrm{AlCl3}$ & $\begin{array}{l}\text { Crocin/ } 4 \text { weeks } 5 \text { or } \\
20 \mathrm{mg} / \mathrm{kg} \\
\text { p.o. }\end{array}$ & MWM, OFT & $\begin{array}{l}\uparrow \text { the circulation around the } \\
\text { periphery, } \downarrow \text { time to locate the } \\
\text { platform, } \uparrow \text { serum levels of A } \beta 1 \text { - } \\
42, \downarrow \text { cerebral cortex levels of A } \beta 1 \text { - } \\
42, \text { deposition of A } \beta 1-42 \text { in the } \\
\text { hippocampal area=> suppressed, } \\
\text { pathological changes of the levels } \\
\text { of Ach, AchE and ChAT in the } \\
\text { serum, cerebral cortex, and } \\
\text { hypothalamus of the AD } \\
\text { mice=>restored, } \uparrow S O D, \uparrow G S H-P x \\
\text { levels in the serum, cerebral } \\
\text { cortex, and hypothalamus }\end{array}$ & [81] \\
\hline Rat/ 37,5 & 6-OHDA & $\begin{array}{l}\text { Crocin alone or } \\
\text { accompanied by } \\
\text { treadmill exercise/ } \\
100 \mathrm{mg} / \mathrm{kg} / \text { i.p. }\end{array}$ & PAT, EPM & $\begin{array}{l}\text { total net No. of rotations } \downarrow \text {, STL } \uparrow \text {, } \\
\text { transfer latency } \downarrow \text {, TNF- } \alpha \text { levels in } \\
\text { the striatum } \downarrow \text {, TBARS levels in } \\
\text { the hippocampus } \downarrow \text {, total thiol } \\
\text { concentration } \uparrow\end{array}$ & {$[82]$} \\
\hline Rat/ 56, 4 & $\begin{array}{l}\text { Trimethylti } \\
\mathrm{n} \text { chloride } \\
\text { (TMT) }\end{array}$ & $\begin{array}{l}\text { Crocin/ } 30 \text { days/ 25, } \\
50 \mathrm{mg} / \mathrm{kg} / \text { i.p. }\end{array}$ & N.M. & $\begin{array}{l}\downarrow \text { Bax, } \downarrow \text { Caspase-9 levels, } \uparrow \text { Bcl- } 2 \text {, } \\
\text { Pt and A } \beta 40 \text { levels } \downarrow, \text { BDNF } \\
\text { levels } \uparrow, \downarrow \text { IL- } 6, \downarrow \text { IL- } 1 \beta, \downarrow \text { TNF- } \alpha \text {, } \\
\uparrow \text { the neuronal density of CA1, } \\
\text { CA } 2 \text {, and CA3 }\end{array}$ & [83] \\
\hline Rat/ 30, 4 & TMT & $\begin{array}{l}\text { Crocin alone or } \\
\text { accompanied by }\end{array}$ & N.M. & IGF1, Glycogen $\uparrow$ & [84] \\
\hline
\end{tabular}




\begin{tabular}{|c|c|c|c|c|c|}
\hline $\begin{array}{l}\text { Animal/ } \\
\text { Sample size } \\
\text { (No. of } \\
\text { groups) }\end{array}$ & $\begin{array}{l}\text { Model of } \\
\text { Study }\end{array}$ & $\begin{array}{l}\text { Targeted compound } \\
\text { /Duration/ } \\
\text { Dosage/ } \\
\text { Route of } \\
\text { Administration }\end{array}$ & $\begin{array}{l}\text { Cognition } \\
\text { Test }\end{array}$ & Major Outcomes & Ref. \\
\hline & & $\begin{array}{l}\text { treadmill exercise/ } 8 \\
\text { weeks } / 25 \mathrm{mg} / \mathrm{kg}\end{array}$ & & & \\
\hline Rat/ 60,5 & $\begin{array}{l}\text { Methamphe } \\
\text { tamine }\end{array}$ & $\begin{array}{l}\text { Crocin/ } 21 \text { days } / 10, \\
\text { 20, 40, and } 80 \\
\text { mg/kg/ i.p. }\end{array}$ & MWM & $\begin{array}{l}\downarrow \mathrm{EL}, \uparrow \mathrm{TSTQ}, \text { swimming } \\
\text { speed=>NSD, } \uparrow \mathrm{GSH}, \downarrow \mathrm{GSSG} \\
\text { levels, } \downarrow \mathrm{MDA}, \uparrow \mathrm{SOD}, \uparrow \mathrm{GSH}-\mathrm{Px}, \\
\uparrow \mathrm{GR}, \downarrow \mathrm{IL}-1 \beta, \downarrow \mathrm{TNF}-\alpha, \uparrow \mathrm{Bcl}-2 \\
\text { expression, } \downarrow \text { Bax protein } \\
\text { expression, } \uparrow \mathrm{BDNF}, \text { and CREB } \\
\text { protein expression }\end{array}$ & {$[85]$} \\
\hline Rat/ 48,4 & $\mathrm{~A} \beta 25-35$ & $\begin{array}{l}\text { crocin } 14 \text { days/ } 40 \\
\mathrm{mg} / \mathrm{kg} / \text { i.p. }\end{array}$ & $\begin{array}{l}\text { Y-maze, } \\
\text { sucrose } \\
\text { preference } \\
\text { test }\end{array}$ & $\begin{array}{l}\downarrow \text { Attempting times, } \uparrow \text { correct } \\
\text { reaction rate, } \uparrow \text { sucrose preference, } \\
\downarrow \text { number of apoptotic cells, } \uparrow \text { Bcl- } \\
2, \downarrow \text { Bax, } \downarrow \text { Caspase- } 3, \downarrow \text { CHOP, } \\
\downarrow \text { GRP78, } \downarrow \text { endoplasmic reticulum } \\
\text { stress in hippocampus and PFC }\end{array}$ & {$[86]$} \\
\hline Rat/ 70, 7 & $\begin{array}{l}\text { methylphen } \\
\text { idate }\end{array}$ & $\begin{array}{l}\text { Crocin/ } 21 \text { days/ 10, } \\
\text { 20, 40, and } 80 \\
\text { mg/kg/ i.p. }\end{array}$ & MWM, OFT & $\begin{array}{l}\downarrow \mathrm{EL} \downarrow \text { traveled distance, } \\
\text { swimming speed=>NSD, } \uparrow \mathrm{TSTQ} \text {, } \\
\uparrow \text { frequency of central square } \\
\text { entries, } \uparrow \text { time spent in the central } \\
\text { region, } \uparrow \text { ambulation distance and } \\
\text { rearing number, } \uparrow \mathrm{GSH} \text { content, } \\
\downarrow \mathrm{GSSG}, \uparrow \mathrm{SOD}, \uparrow \mathrm{GSH}-\mathrm{Px}, \text { and } \\
\uparrow \mathrm{GR}, \downarrow \mathrm{MDA}, \downarrow \mathrm{IL}-1 \beta, \downarrow \mathrm{TNF}-\alpha, \\
\uparrow \mathrm{Bcl}-2, \downarrow \text { Bax protein expression, } \\
\uparrow \text { protein expression in BDNF and } \\
\text { CREB total and phosphorylated } \\
\text { forms }\end{array}$ & {$[87]$} \\
\hline Rat/ 66, 6 & $\mathrm{~A} \beta 1-40$ & $\begin{array}{l}\text { Safranal/ } 7 \text { days/ } \\
0.025,0.1 \text {, or } 0.2 \\
\mathrm{ml} / \mathrm{kg} / \mathrm{p} .0 .\end{array}$ & $\begin{array}{l}\text { Y-maze, } \\
\text { NORT, } \\
\text { RAM, PAT }\end{array}$ & $\begin{array}{l}\text { reduction of alternation score }=> \\
\text { prevented, } \uparrow \text { discrimination ratio, } \\
\uparrow \mathrm{STL}, \mathrm{IL}=>\mathrm{NSD}, \downarrow \text { working } \\
\text { memory errors, } \downarrow \text { reference } \\
\text { memory errors, } \downarrow \text { MDA, } \downarrow \text { Nitrite, } \\
\downarrow \text { protein carbonyl, } \downarrow \text { ROS, } \uparrow \mathrm{CAT}, \\
\uparrow \mathrm{SOD}, \uparrow \mathrm{GSH}, \downarrow \mathrm{IL}-1 \beta, \downarrow \mathrm{IL}-6, \\
\downarrow \mathrm{TNF} \alpha, \downarrow N F-\mathrm{kB}, \downarrow \text { caspase } 3 \\
\text { activity, } \downarrow \mathrm{DNA} \text { fragmentation, } \\
\downarrow \text { GFAP in the hippocampus, } \\
\downarrow \text { AChE activity, } \uparrow \mathrm{MMP}, \downarrow \text { number } \\
\text { of CA } 1 \text { neurons }\end{array}$ & {$[88]$} \\
\hline Rat/ 48,5 & TMT & $\begin{array}{l}\text { Crocin alone or } \\
\text { accompanied by } \\
\text { treadmill exercise/ } 8 \\
\text { weeks } / 25 \mathrm{mg} / \mathrm{kg} / \text { i.p. }\end{array}$ & EPM & $\begin{array}{l}\downarrow \text { weight levels, } \uparrow \% \text { Open Arm } \\
\text { Entry, } \uparrow \% \text { Elapsed Time in the } \\
\text { Open Arm, } \uparrow \text { Aerobic Power }\end{array}$ & [89] \\
\hline Rat/ 48,6 & $\begin{array}{l}\text { Post- } \\
\text { traumatic } \\
\text { stress } \\
\text { disorder }\end{array}$ & $\begin{array}{l}\text { Saffron and Crocin/ } \\
28 \text { days/ } 10 \mu \mathrm{g} / \mathrm{ICV}\end{array}$ & $\begin{array}{l}\text { Barnes } \\
\text { Maze }\end{array}$ & $\begin{array}{l}\uparrow \text { corticosterone concentration, } \\
\downarrow \text { Freezing Behavior, Search } \\
\text { Strategies (direct, random and } \\
\text { serial)=>NSD, } \downarrow \text { No. of Errors in } \\
\text { finding the target hole, } \downarrow \text { Traveled } \\
\text { Distance, Latency Time }=>\text { NSD }\end{array}$ & [90] \\
\hline Rat/ 36, 6 & Hyoscine & $\begin{array}{l}\text { Crocin/ } 5 \text { days/ 10, } \\
\text { 20, and } 40 \mathrm{mg} / \mathrm{kg} / \\
\text { i.p. }\end{array}$ & MWM & $\begin{array}{l}\downarrow E L, \uparrow T S T Q, \text { swimming } \\
\text { speed }=>\text { NSD, NMDA and AMPA } \\
\text { protein expression =>NSD, } \\
\text { CaMKII, pCaMKII, and ERK } \\
\text { proteins }=>\text { NSD, a decrease of } \\
\text { pERK protein level=>inhibited, } \\
\text { ERK, CaMKII, NMDA and } \\
\text { AMPA RNA expression=> NSD }\end{array}$ & {$[91]$} \\
\hline Rat/ 30, 5 & hypoxia & $\begin{array}{l}\text { Crocin/ } 3 \text { days/ } 25, \\
50,100 \mathrm{mg} / \mathrm{kg} / \mathrm{i} . \mathrm{m} .\end{array}$ & MWM & $\begin{array}{l}\downarrow \mathrm{EL}, \uparrow \mathrm{TSTQ}, \uparrow \text { No. of crossings, } \\
\uparrow \text { mitochondria of nerve cells, } \\
\text { mitochondria swelling and cristae } \\
\text { blurring=>improved, } \uparrow \text { SIRT } 1 \text { and } \\
\text { PGC- } 1 \alpha \text { proteins distribution, } \uparrow \text { the } \\
\text { hippocampal density of SIRT1 and } \\
\text { PGC- } 1 \alpha\end{array}$ & {$[92]$} \\
\hline
\end{tabular}




\begin{tabular}{|c|c|c|c|c|c|}
\hline $\begin{array}{l}\text { Animal/ } \\
\text { Sample size } \\
\text { (No. of } \\
\text { groups) }\end{array}$ & $\begin{array}{l}\text { Model of } \\
\text { Study }\end{array}$ & $\begin{array}{l}\text { Targeted compound } \\
\text { /Duration/ } \\
\text { Dosage/ } \\
\text { Route of } \\
\text { Administration }\end{array}$ & $\begin{array}{l}\text { Cognition } \\
\text { Test }\end{array}$ & Major Outcomes & Ref. \\
\hline Mouse/ 26, 3 & APPsw & $\begin{array}{l}\text { Crocetin/ } 6 \text { months/ } \\
10,30 \mathrm{mg} / \mathrm{kg} / \text { p.o. }\end{array}$ & $\begin{array}{l}\text { MWM, } \\
\text { NORT }\end{array}$ & 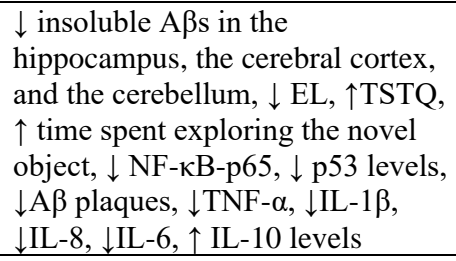 & [93] \\
\hline Rat/ 40, 5 & morphine & $\begin{array}{l}\text { Crocin/ } 21 \text { days/ } \\
12.5,25 \mathrm{mg} / \mathrm{kg} / \text { i.p. }\end{array}$ & N.M. & $\begin{array}{l}\downarrow \text { glucose level, serum } \\
\text { cholesterol=>NSD, serum } \\
\text { triglyceride level=>NSD, } \uparrow \text { BDNF } \\
\text { and CREB gene expression in the } \\
\text { VTA area, } \uparrow \text { serum BDNF level }\end{array}$ & [94] \\
\hline Rat/ 32, 4 & normal & $\begin{array}{l}\text { Crocins alone and in } \\
\text { combination with } \\
\text { memantine/ } 5,15 \text {, } \\
\text { and } 30 \mathrm{mg} / \mathrm{kg} / \text { i.p. }\end{array}$ & NORT & $\begin{array}{l}\uparrow \text { Discrimination (D) index, Total } \\
\text { exploration times=>NSD, Total } \\
\text { motor activity=>NSD }\end{array}$ & [95] \\
\hline Rat/ 40, 5 & $\begin{array}{l}\text { isolation } \\
\text { stress }\end{array}$ & $\begin{array}{l}\text { Crocin/ } 21 \text { days/ } 30 \\
\text { and } 60 \mathrm{mg} / \mathrm{kg} / \text { i.p. }\end{array}$ & $\begin{array}{l}\text { NOLT, } \\
\text { NORT }\end{array}$ & $\begin{array}{l}\text { OLT; } \uparrow \text { Total time of object } \\
\text { exploration, } \uparrow \text { novel object } \\
\text { exploration time, } \uparrow \text { Values of the } \\
\text { main discrimination index (D2), } \\
\uparrow \text { values of auxiliary discrimination } \\
\text { index (D1) / NORT; } \uparrow T \text { Tatal time } \\
\text { of object exploration, } \uparrow \text { novel } \\
\text { object exploration time, } \uparrow \text { Values } \\
\text { of the main discrimination index } \\
\text { (D2), } \uparrow \text { values of auxiliary } \\
\text { discrimination index (D1), serum } \\
\text { corticosterone levels }\end{array}$ & [96] \\
\hline Rat/ 40, 5 & $\begin{array}{l}\text { A } \beta \text { peptide } \\
(1-42)\end{array}$ & $\begin{array}{l}\text { Crocin/12 days/ } \\
30 \mathrm{mg} / \mathrm{kg} / \text { i.p. }\end{array}$ & $\begin{array}{l}\text { Barnes } \\
\text { maze, PAT }\end{array}$ & $\begin{array}{l}\downarrow \text { latency time to achieve the target } \\
\text { hole, } \downarrow \text { number of errors to find the } \\
\text { target hole, } \uparrow \text { STL, } \downarrow \text { c-Fos, } \\
\downarrow \text { number of TUNEL-positive cells } \\
\text { in the CA } 1 \text { region, cell } \\
\text { death=>prevented, } \uparrow P S \text { amplitude, } \\
\downarrow \text { fEPSP slope, LTP } \\
\text { induction=> prevented }\end{array}$ & {$[97]$} \\
\hline Rat/ 88,8 & $\begin{array}{l}\text { streptozotoc } \\
\text { in } \\
\text { (STZ) }\end{array}$ & $\begin{array}{l}\text { Safranal alone and in } \\
\text { combinnation with } \\
\text { metformin/ } 37 \text { days/ } \\
0.025,0.1 \text { and } 0.4 \\
\mathrm{mg} / \mathrm{kg} / \text { i.p. }\end{array}$ & MWM & $\begin{array}{l}\downarrow \text { hyperglycemia, } \downarrow \text { EL, } \uparrow \text { TSTQ, } \\
\downarrow \text { distance swam, hippocampal } \\
\text { neuron loss }=>\text { recovered, } \downarrow \text { MDA, } \\
\downarrow \text { TNF- } \alpha, \downarrow \text { Caspase- } 3, \uparrow \text { SOD }\end{array}$ & {$[98]$} \\
\hline Rat/ 48,6 & $\begin{array}{l}\text { Chronic } \\
\text { unpredictab } \\
\text { le Stress }\end{array}$ & $\begin{array}{l}\text { Crocin/ } 21 \text { days/ } 30 \\
\mathrm{mg} / \mathrm{kg} / \text { i.p. }\end{array}$ & PAT & $\begin{array}{l}\uparrow \mathrm{STL}, \uparrow \text { IL to enter the darkroom, } \\
\text { total dark compartment stay } \\
\text { time=>NSD }\end{array}$ & [99] \\
\hline Mouse, rat & $\begin{array}{l}\text { 5XFAD, } \\
\text { STZ, } \\
\text { scopolamin } \\
\text { e }\end{array}$ & $\begin{array}{l}\text { IIIM-141 trans-4- } \\
\text { GG-crocin }(36 \% \\
\text { w/w)/ } 28 \text { days } / 12.5 \text {, } \\
25,50,100 \mathrm{mg} / \mathrm{kg} / \\
\text { p.o. }\end{array}$ & $\begin{array}{l}\text { MWM } \\
\text { (STZ-rat), } \\
\text { PAT } \\
\text { (Scopopola } \\
\text { mine- } \\
\text { mouse) } \\
\end{array}$ & $\begin{array}{l}\text { MWM; } \downarrow \text { transfer latency time, } \downarrow \% \\
\text { change in transfer latency } \\
\text { PAT; } \uparrow \text { transfer latency time, } \uparrow \% \\
\text { change in transfer latency }\end{array}$ & [100] \\
\hline Rat/ 35,5 & Tramadol & $\begin{array}{l}\text { Crocin/ } 28 \text { day/ } 30 \\
\mathrm{mg} / \mathrm{kg} / \text { p.o. }\end{array}$ & MWM, PAT & $\begin{array}{l}\text { TSTQ }=>\text { NSD, } \uparrow \text { traveled distance, } \\
\uparrow \text { time delay for entering the dark } \\
\text { compartment, } \downarrow \text { total time spent in } \\
\text { the dark compartment, No. of } \\
\text { entries to the dark } \\
\text { compartment }=>\text { NSD, } \uparrow \text { total time } \\
\text { spent in the light compartment, } \\
\downarrow \text { No. of TUNEL positive cells in } \\
\text { CA1, CA3 and DG of the } \\
\text { hippocampus, DNS No. per unit } \\
\text { area in CA3 and DG of the } \\
\text { hippocampus }\end{array}$ & [101] \\
\hline $\begin{array}{l}\text { Rat/ } \\
\text { NORT; 60/ } \\
\text { NOLT; 48 }\end{array}$ & $\begin{array}{l}\text { Apomorphi } \\
\text { ne }\end{array}$ & $\begin{array}{l}\text { Crocins/ } 3 \text { days/ } 15 \\
\text { and } 30 \mathrm{mg} / \mathrm{kg} / \mathrm{i} . \mathrm{p} .\end{array}$ & $\begin{array}{l}\text { NOLT, } \\
\text { NORT }\end{array}$ & $\begin{array}{l}\text { NORT; } \uparrow \text { index D, exploration } \\
\text { times }=>\text { NSD, NOLT; D } \\
\text { index=>NSD, exploration }\end{array}$ & [102] \\
\hline
\end{tabular}




\begin{tabular}{|c|c|c|c|c|c|}
\hline $\begin{array}{l}\text { Animal/ } \\
\text { Sample size } \\
\text { (No. of } \\
\text { groups) }\end{array}$ & $\begin{array}{l}\text { Model of } \\
\text { Study }\end{array}$ & $\begin{array}{l}\text { Targeted compound } \\
\text { /Duration/ } \\
\text { Dosage/ } \\
\text { Route of } \\
\text { Administration }\end{array}$ & $\begin{array}{l}\text { Cognition } \\
\text { Test }\end{array}$ & Major Outcomes & Ref. \\
\hline & & & & $\begin{array}{l}\text { times }=>N S D \text {, Total motor } \\
\text { activity }=>\text { NSD }\end{array}$ & \\
\hline Mouse/ 40, 5 & $\begin{array}{l}\text { pentylenetet } \\
\text { razol }\end{array}$ & $\begin{array}{l}\text { Crocin/ } 36 \text { days/ 5, } \\
10 \text { and } 20 \mathrm{mg} / \mathrm{kg} / \mathrm{p} . \mathrm{o} .\end{array}$ & $\begin{array}{l}\text { T-maze, } \\
\text { NORT }\end{array}$ & $\begin{array}{l}\downarrow \text { Seizure severity score, } \\
\uparrow(\%) \text { spontaneous alternation, } \\
\uparrow(\%) \text { preference index, } \uparrow \\
\text { discrimination ratio, } \uparrow \text { SOD, } \\
\downarrow \text { number of Nissl-stained dark } \\
\text { neurons, } \downarrow \text { NF-KB expression, } \downarrow \text { p- } \\
\text { NF-KB expression }\end{array}$ & [103] \\
\hline Mouse/ 32, 4 & $\begin{array}{l}\text { aflatoxin } \\
\text { B1 }\end{array}$ & $\begin{array}{l}\text { Saffron tea/ } 2 \text { weeks } \\
90 \mathrm{mg} \text { styles/200 mL } \\
(3.6 \mathrm{mg} \text { saffron/ } \\
\text { mouse }) / \text { p.o. }\end{array}$ & PAT & $\begin{array}{l}\text { body weight gain, food and liquid } \\
\text { intake=>NSD, } \uparrow \text { STL, IL=>NSD, } \\
\downarrow \text { SS- and DS-AChE activity in } \\
\text { whole brain and cerebellum, } \uparrow \text { the } \\
\text { activity of whole-brain DS-BuChE } \\
\text { was at control levels, } \downarrow \text { liver SS- } \\
\text { AChE and BuChE activity, } \downarrow \text { liver } \\
\text { DS-BuChE, } \downarrow \text { MAO-A and MAO- } \\
\text { B, } \downarrow \text { MDA in the whole brain, } \\
\text { cerebellum, and liver, } \downarrow \text { GSH } \\
\text { values in brain, cerebellum, and } \\
\text { liver }\end{array}$ & [104] \\
\hline Rat/ 30, 6 & D-galactose & $\begin{array}{l}\text { Crocin/56 days/ 7.5, } \\
15,30 \mathrm{mg} / \mathrm{kg} / \mathrm{i} . \mathrm{p} .\end{array}$ & MWM & $\begin{array}{l}\downarrow \mathrm{EL}, \uparrow \mathrm{TSTQ}, \text { swimming } \\
\text { speeds }=>\mathrm{NSD}, \downarrow \text { MDA, } \downarrow \mathrm{CML} \\
\text { expression, } \uparrow \text { phosphorylation of } \\
\text { Akt protein, MAPK activity } \\
\text { through the elevation of pErk/Erk } \\
\text { ratio } \uparrow, \downarrow N F-\kappa B \text { p65 protein level } \\
\text { in the hippocampus, } \downarrow \text { IL-1 } \beta \text { and } \\
\text { TNF } \alpha \text { formations }\end{array}$ & [105] \\
\hline Rat/ 40, 5 & $\begin{array}{l}\text { Chronic } \\
\text { Restraint } \\
\text { Stress } \\
\end{array}$ & $\begin{array}{l}\text { Crocin/ } 21 \text { days/ } 30 \\
\text { and } 60 \mathrm{mg} / \mathrm{kg} / \text { i.p. }\end{array}$ & PAT & $\begin{array}{l}\uparrow \mathrm{STL}, \uparrow \mathrm{IL}, \downarrow \text { corticosterone levels } \\
\text { in the hippocampus and frontal } \\
\text { cortex }\end{array}$ & [106] \\
\hline Rat/ 60, 6 & $\mathrm{~A} \beta(25-35)$ & $\begin{array}{l}\text { Saffron / } 21 \text { days/ } 5 \text {, } \\
10 \text { mg/kg/ i.p. }\end{array}$ & $\begin{array}{l}\text { The Y-maze } \\
\text { task, RAM }\end{array}$ & $\begin{array}{l}\text { In vitro antioxidant; } \uparrow \text { DPPH } \\
\text { scavenging, ABTS } \bullet+\text { scavenging } \\
\text { (general experimental tests)/ In } \\
\text { vivo; } \uparrow \text { short working memory, } \\
\uparrow(\%) \text { spontaneous alternation, } \uparrow \\
\text { No. of arms entries, } \downarrow \text { Working } \\
\text { memory errors, } \downarrow \text { reference } \\
\text { memory errors }\end{array}$ & [107] \\
\hline Rat/ 36, 6 & hyoscine & $\begin{array}{l}\text { Crocin/ } 5 \text { days/ 10, } \\
20,40 \mathrm{mg} / \mathrm{kg} / \text { i.p. }\end{array}$ & MWM & $\begin{array}{l}\uparrow T S T Q, \text { Swim Speed in the Target } \\
\text { Quadrant and Total Swim } \\
\text { Speed=>NSD, } \downarrow \text { Latency Time to } \\
\text { Find Platform, } \uparrow \text { BDNF, } \uparrow \text { p- } \\
\text { CREB, } \uparrow \text { CREB }\end{array}$ & [108] \\
\hline Mouse/ 18, 2 & 5XFAD & $\begin{array}{l}1 \mathrm{month} / \text { Saffron; } 50 \\
\mathrm{mg} / \mathrm{kg} / \text { Crocin; } 10 \\
\mathrm{mg} / \mathrm{kg} / \mathrm{p} . \mathrm{o} .\end{array}$ & N.M. & $\begin{array}{l}\downarrow \text { total } A \beta \text { and } A \beta \text { o levels in the } \\
\text { brain, } \downarrow \text { levels of monomeric } A \beta 40 \\
\text { and } A \beta 42 \text { in the brain, } \uparrow \text { LRP } 1 \text { and } \\
\text { P-gp expressions, expression of } \\
\text { the } A \beta \text { degrading enzyme NEP in } \\
\text { brain homogenates } \uparrow \text {, expression of } \\
\text { IDE levels }=>\text { NSD, } \uparrow \text { ABCA } 1 \\
\text { expression, } \uparrow \text { PPAR } \gamma \text { expression, } \\
\downarrow \text { IgG extravasation, } \uparrow \text { claudin-5 } \\
\text { expression in brain microvessels, } \\
\uparrow \text { PSD-95 expression, } \uparrow \text { SNAP- } 25 \\
\text { expression, } \downarrow \text { brain levels of IL-1 } 1 \beta \text {, } \\
\downarrow \text { GFAP optical intensity }\end{array}$ & [109] \\
\hline Rat/ 35,5 & $\begin{array}{l}\text { streptozotoc } \\
\text { in }\end{array}$ & $\begin{array}{l}\text { Crocin/ } 6 \text { weeks/ 15, } \\
30 \text { and } 60 \mathrm{mg} / \mathrm{kg} / \text { i.p. }\end{array}$ & MWM & $\begin{array}{l}\downarrow \text { serum glucose levels, } \downarrow \text { EL, } \\
\downarrow \text { traveled distance, } \uparrow \text { TSTQ, } \\
\downarrow \text { TBARS level in the cortex, total } \\
\text { thiol concentration in the } \\
\text { cortex }=>\text { NSD }\end{array}$ & [110] \\
\hline
\end{tabular}




\begin{tabular}{|c|c|c|c|c|c|}
\hline $\begin{array}{l}\text { Animal/ } \\
\text { Sample size } \\
\text { (No. of } \\
\text { groups) }\end{array}$ & $\begin{array}{l}\text { Model of } \\
\text { Study }\end{array}$ & $\begin{array}{l}\text { Targeted compound } \\
\text { /Duration/ } \\
\text { Dosage/ } \\
\text { Route of } \\
\text { Administration }\end{array}$ & $\begin{array}{l}\text { Cognition } \\
\text { Test }\end{array}$ & Major Outcomes & Ref. \\
\hline Rat/ 60 & ketamine & $\begin{array}{l}\text { Crocin/ } / 2,5 \text { or } 10 \\
\mathrm{mg} / \mathrm{kg} / \text { i.p. }\end{array}$ & PAT & $\begin{array}{l}\text { STL=>NSD, the performance of } \\
\text { the rats in the shuttle } \\
\text { box=>improved }\end{array}$ & [111] \\
\hline Rat/ 32,4 & 6-OHDA & $\begin{array}{l}\text { Crocin/ } 6 \text { weeks } / 30 \\
\text { and } 60 \mathrm{mg} / \mathrm{kg} / \text { i.p. }\end{array}$ & PAT & $\begin{array}{l}\downarrow \text { TBARS levels in the } \\
\text { hippocampus, } \downarrow \text { nitrite levels in the } \\
\text { hippocampus, total thiol } \\
\text { concentrations } \\
\text { in the hippocampus and } \\
\text { cortex=>NSD, GSH-Px=>NSD, } \\
\uparrow S T L\end{array}$ & [112] \\
\hline Rat/ 10,2 & aging & $\begin{array}{l}\text { safranal /30 days/ } 0.5 \\
\mathrm{mg} / \mathrm{kg} / \text { i.p. }\end{array}$ & N.M. & $\downarrow \mathrm{MDA}, \uparrow \mathrm{GSH}-\mathrm{Px}, \uparrow \mathrm{SOD}, \uparrow \mathrm{GST}$ & [113] \\
\hline Rat/ 24,4 & acrolein & $\begin{array}{l}\text { Crocin/ } 2 \text { weeks/ } \\
12.5,25,50 \\
\text { mg/kg/day/ i.p. }\end{array}$ & N.M. & $\begin{array}{l}\downarrow \text { MDA, } \uparrow \text { GSH-Px, } \downarrow \text { A } \beta 1- \\
42, \downarrow T 231 \text { phosphorylation state, } \\
\downarrow \text { phosphorylation of Ser396, } \downarrow \text { p- } \\
\text { Akt, levels phosphorylated and } \\
\text { total GSK-3 } \beta \text { at Ser9 GSK- } \\
3 \beta=>\text { NSD }, \downarrow \text { p-ERK } 1 / 2, \downarrow \text { p-JNK, } \\
\text { level of p-p38=>NSD }\end{array}$ & [114] \\
\hline Rat/ 40,5 & $\begin{array}{l}\text { ethidium } \\
\text { bromide }\end{array}$ & $\begin{array}{l}\text { Saffron/1 week/ } 5 \\
\text { and } 10 \mu \mathrm{g} / \text { i.p. }\end{array}$ & MWM & $\begin{array}{l}\downarrow E L, \uparrow \text { traveled the distance to find } \\
\text { the platform, } \downarrow \text { FRAP value, } \\
\downarrow \text { TBARS levels, } \downarrow \text { GSH-Px } \\
\text { activity, } \uparrow \text { SOD }\end{array}$ & [115] \\
\hline Rat & $\mathrm{A} \beta(1-42)$ & $\begin{array}{l}\text { Crocin/ } 21 \text { days/i.h.; } \\
\text { 150, } 300,600 \mathrm{nmol} / \\
\text { i.p.; } 30 \mathrm{mg} / \mathrm{kg}\end{array}$ & MWM & $\begin{array}{l}\uparrow \text { traveled distance, } \downarrow \text { EL, } \\
\text { swimming speed=>NSD, } \uparrow \text { TSTQ, } \\
\text { Beclin-1=>NSD, LC3-II/LC3-I } \\
\text { ratio=>NSD, } \downarrow \text { Bax, } \uparrow \text { Bcl-2, } \\
\downarrow \text { Bax/Bcl-2 ratio, } \downarrow \text { Caspase-3 }\end{array}$ & [116] \\
\hline Rat/ 50, 5 & STZ & $\begin{array}{l}\text { Saffron/ } 4 \text { weeks/ 20, } \\
\text { 40, and } 80 \mathrm{mg} / \mathrm{kg} / \text { i.p. }\end{array}$ & MWM & $\begin{array}{l}\uparrow \text { Bodyweight, } \downarrow \text { Blood Glucose } \\
\text { Levels, } \downarrow \text { EL, } \uparrow \text { TSTQ, } \downarrow \text { TNF } \alpha, \\
\downarrow \text { serum levels of total Lipids, } \\
\downarrow \text { triglycerides, } \downarrow \text { total cholesterol, } \\
\downarrow \text { LDL, } \uparrow \text { HDL, } \downarrow \text { GSP levels, } \\
\downarrow \text { Serum AGEs, } \uparrow \text { GSH, } \uparrow \text { SOD, } \\
\uparrow \text { CAT, } \downarrow \text { iNOS }\end{array}$ & [117] \\
\hline Rat/ 20, 4 & STZ & $\begin{array}{l}\text { Crocin/21 days/ } 100 \\
\mathrm{mg} / \mathrm{kg} / \text { p.o. }\end{array}$ & N.M. & $\begin{array}{l}\downarrow \text { MDA levels in the striatum, } \\
\uparrow \text { total thiol, } \uparrow \text { GSH-Px activity }\end{array}$ & [118] \\
\hline Rat/ 60, 6 & ketamine & $\begin{array}{l}\text { Crocins/ } 3 \text { days/ 30, } \\
50 \mathrm{mg} / \mathrm{kg} / \text { i.p. }\end{array}$ & NORT & $\begin{array}{l}\uparrow \text { discrimination index D, } \downarrow \text { ataxia, } \\
\text { total object exploration } \\
\text { times=>NSD }\end{array}$ & [119] \\
\hline Rat/ 30, 5 & ketamine & $\begin{array}{l}\text { Crocetin/ } 7 \text { days/ 2,4, } \\
8 \mathrm{mg} / \mathrm{kg} / \text { i.p. }\end{array}$ & MWM & $\begin{array}{l}\downarrow \mathrm{EL}, \uparrow \mathrm{TSTQ}, \text { swimming } \\
\text { speed=>NSD, the morphology of } \\
\text { neurons in cortex and } \\
\text { hippocampus CA1, CA2, CA3 } \\
\text { regions=>protected }\end{array}$ & [120] \\
\hline $\begin{array}{l}\text { Rat/ 48, } 8 \\
\text { groups }\end{array}$ & STZ & $\begin{array}{l}\text { Crocin/ } 27 \text { days/ 7.5, } \\
15 \text { and } 30 \mathrm{mg} / \mathrm{kg} / \text { i.p. }\end{array}$ & EPM & $\begin{array}{l}\uparrow \text { Transfer latency, } \downarrow \text { Blood glucose, } \\
\uparrow \text { serum insulin, } \uparrow \text { TAC, } \downarrow \text { MDA, a } \\
\text { decrease of neurons } \\
\text { in the hippocampus }=>\text { prevented }\end{array}$ & [121] \\
\hline Rat/ 35,5 & $\begin{array}{l}\text { Quinolinic } \\
\text { Acid }\end{array}$ & $\begin{array}{l}\text { Safranal/ } 72.75, \\
\text { 145.5, and } 291 \\
\text { mg/kg/i.p. }\end{array}$ & N.M. & $\begin{array}{l}\text { (general antioxidant tests were } \\
\text { been assessed in vitro), } \downarrow \text { MDA, } \\
\uparrow \text { antioxidant power (FRAP value) } \\
\text { of brain homogenate samples, } \uparrow \\
\text { total thiol content, } \downarrow \text { DNA damage }\end{array}$ & [122] \\
\hline Mouse/ 30, 3 & $\mathrm{AlCl} 3$ & $\begin{array}{l}\text { Saffron/ } 6 \text { days/ } 60 \\
\mathrm{mg} / \mathrm{kg} / \text { i.p. }\end{array}$ & PAT & $\begin{array}{l}\text { Step through latency }=>N S D \text {, } \\
\text { Initial latency }=>N S D, \downarrow S S-\text { and } \\
\text { DS-AChE activity in whole brain } \\
\text { and cerebellum, cerebral } \\
\text { BuChE }=>\text { NSD, } \downarrow \text { SS- and DS- } \\
\text { liver BuChE and SS-AChE, } \downarrow \\
\text { MAO-A and MAO-B activity of } \\
\text { the whole brain, } \uparrow \mathrm{MAO}-\mathrm{B} \text { activity } \\
\text { of cerebellum, } \downarrow \mathrm{MDA}, \uparrow \text { GSH }\end{array}$ & [123] \\
\hline
\end{tabular}




\begin{tabular}{|c|c|c|c|c|c|}
\hline $\begin{array}{l}\text { Animal/ } \\
\text { Sample size } \\
\text { (No. of } \\
\text { groups) }\end{array}$ & $\begin{array}{l}\text { Model of } \\
\text { Study }\end{array}$ & $\begin{array}{l}\text { Targeted compound } \\
\text { /Duration/ } \\
\text { Dosage/ } \\
\text { Route of } \\
\text { Administration }\end{array}$ & $\begin{array}{l}\text { Cognition } \\
\text { Test }\end{array}$ & Major Outcomes & Ref. \\
\hline Mouse/ 24, 3 & Morphine & $\begin{array}{l}\text { Saffron/ } 3 \text { days/ } 50 \text {, } \\
150 \text {, and } 450 \mathrm{mg} / \mathrm{kg} / \\
\text { i.p. }\end{array}$ & PAT & $\begin{array}{l}\uparrow \text { total time spent in the light } \\
\text { compartment, } \downarrow \text { total time spent in } \\
\text { the dark compartment }\end{array}$ & {$[124]$} \\
\hline Rat/ 32,4 & STZ & $\begin{array}{l}\text { Crocetin in a } \\
\text { combination/ } 15 \\
\text { days } / 25 \mu \mathrm{g} / \mathrm{kg} / \mathrm{p} . \mathrm{o} .\end{array}$ & MWM, PAT & $\begin{array}{l}\uparrow \mathrm{STL}, \mathrm{IL}=>\mathrm{NSD}, \downarrow \mathrm{EL}, \uparrow \mathrm{TSTQ}, \\
\downarrow \mathrm{TBARS} \text { level in rat hippocampus } \\
\text { and frontal cortex, } \uparrow \mathrm{GSH}, \uparrow \mathrm{GPx}, \\
\uparrow \mathrm{GST}, \uparrow \mathrm{SOD}, \uparrow \mathrm{CAT}\end{array}$ & [125] \\
\hline Rat/ 70, 8 & $\begin{array}{l}\text { chronic } \\
\text { cerebral } \\
\text { hypoperfusi } \\
\text { on } \\
\end{array}$ & $\begin{array}{l}5 \text { days/ saffron }(50, \\
100 \text { and } 250 \mathrm{mg} / \mathrm{kg}, \\
\text { crocin }(5,10, \\
25 \mathrm{mg} / \mathrm{kg} / \text { i.p. }\end{array}$ & MWM & $\downarrow \mathrm{EL}, \uparrow \mathrm{TSTQ}$ & [126] \\
\hline mouse & $\begin{array}{l}\text { D-galactose } \\
\text { and NaNO2 }\end{array}$ & $\begin{array}{l}\text { Saffron/ } 15 \text { days/ } 30 \\
\mathrm{mg} / \mathrm{kg} / \text { i.p. }\end{array}$ & AAT, PAT & $\uparrow$ avoidance responses, $\uparrow$ STL & [127] \\
\hline Mouse/ 39, 4 & APP/PS1 & $\begin{array}{l}\text { Saffron in } \\
\text { combination/ } 3 \\
\text { months/ 30, } 44 \\
\mathrm{mg} / \mathrm{kg} / \mathrm{p} . \mathrm{o}\end{array}$ & MWM & $\begin{array}{l}\downarrow \mathrm{EL}, \uparrow \mathrm{TSTQ} \text {, cytoplasmic and } \\
\text { nuclear membranes=>NSD, } \\
\uparrow \text { cytoplasmic swelling and } \\
\text { vacuolation, } \downarrow \text { A } \beta \\
\text { immunoreactivity, deformed } \\
\text { mitochondrion detected with } \\
\text { cristae residues, rare } \\
\text { autophagosomes, and dilated } \\
\text { Golgi complex and reticulum }\end{array}$ & [128] \\
\hline Mouse/ 20, 4 & $\begin{array}{l}\mathrm{AlCl} 3 \\
\mathrm{Balb} / \mathrm{c} \text { and } \\
\mathrm{C} 57 \mathrm{BL} / 6\end{array}$ & $\begin{array}{l}\text { Saffron/ } 45 \text { days/ } 200 \\
\text { mg/kg/i.p. }\end{array}$ & N.M. & $\begin{array}{l}\uparrow \mathrm{SOD}, \uparrow \mathrm{CAT}, \uparrow \mathrm{GSH}-\mathrm{Px}, \uparrow \text { Total } \\
\text { antioxidants capacity, } \downarrow \text { TBARS, } \\
\downarrow \text { Arginase, } \downarrow \text { a-l-fucosidase, } \uparrow \mathrm{R}- \\
\text { spondin gene, inositol } \\
\text { polyphosphate phosphatase-like } \\
\text { gene, Bcl- } 2=>\text { abscent }\end{array}$ & [129] \\
\hline Mouse/ 16, 2 & $\begin{array}{l}\text { Balb-c, } \\
\text { aging }\end{array}$ & $\begin{array}{l}\text { Saffron/ } 6 \text { days/ } \\
60 \mathrm{mg} / \mathrm{kg} / \text { i.p. }\end{array}$ & PAT & $\begin{array}{l}\mathrm{IL}=>\mathrm{NSD}, \uparrow \mathrm{STL}, \downarrow \mathrm{SS} \text { and DS } \\
\text { AChE-specific activity, } \downarrow \text { MDA, } \uparrow \\
\text { GSH, } \downarrow \text { caspase-3 }\end{array}$ & {$[130]$} \\
\hline Rat/ 150,8 & $\begin{array}{l}\text { chronic } \\
\text { stress }\end{array}$ & $\begin{array}{l}21 \text { days/ Crocin; } 15, \\
30 \mathrm{mg} / \mathrm{kg} \text {, Saffron; } \\
30 \mathrm{mg} / \mathrm{kg} \text { / i.p. }\end{array}$ & MWM & $\begin{array}{l}\downarrow \text { corticosterone levels, } \downarrow \text { EL, } \\
\uparrow \text { TSTQ, swimming speed=>NSD, } \\
\uparrow \text { FRAP levels, } \downarrow \text { TBARS levels, } \\
\downarrow \text { SOD }, \downarrow \text { GSH }, \downarrow \text { GSH-Px }\end{array}$ & [131] \\
\hline Rat/ 45,3 & STZ & $\begin{array}{l}\text { Saffron/ } 3 \text { weeks/ } 30 \\
\text { mg/kg/ i.p. }\end{array}$ & PAT & $\begin{array}{l}\downarrow I L, \uparrow S T L, \text { patohistolocal } \\
\text { effects }=>\text { NSD }\end{array}$ & [132] \\
\hline Rat/ 90, 6 & STZ & $\begin{array}{l}\text { Crocin } / 2 \text { days/ } 15 \\
\text { and } 30 \mathrm{mg} / \mathrm{kg} / \text { i.p. }\end{array}$ & $\begin{array}{l}\text { Y-maze, } \\
\text { PAT }\end{array}$ & $\begin{array}{l}\downarrow \text { body weight, } \uparrow \text { step-through } \\
\text { latency, } \uparrow(\%) \text { Alternation behavior, } \\
\uparrow \text { No. of animals falling }\end{array}$ & [133] \\
\hline Rat/ 60,5 & STZ & $\begin{array}{l}\text { Saffron/ } 3 \text { weeks/ } \\
60 \mathrm{mg} / \mathrm{kg} / \text { i.p. }\end{array}$ & $\begin{array}{l}\text { Y-maze, } \\
\text { PAT }\end{array}$ & $\begin{array}{l}\downarrow \mathrm{IL}, \uparrow \mathrm{STL}, \uparrow(\%) \text { scores of } \\
\text { Alternation behavior, } \\
\uparrow \text { Psychomotor coordination } \\
\text { (PMC) index }\end{array}$ & [134] \\
\hline $\begin{array}{l}\text { Rat/NORT;4 } \\
0,4 / \mathrm{PAT} ; 80 \\
8\end{array}$ & $\begin{array}{l}\text { scopolamin } \\
\text { e }\end{array}$ & $\begin{array}{l}\text { Saffron/ 10, 30, } 60 \\
\text { mg/kg/i.p. }\end{array}$ & NORT, PAT & $\begin{array}{l}\text { Total motor activity }=>N S D, \text { Total } \\
\text { exploration time }=>\text { NSD, } \\
\uparrow \text { exploration time of the novel } \\
\text { object, } \uparrow \text { Discrimination index D, } \\
\uparrow \text { Retention latencies to enter the } \\
\text { dark chamber }\end{array}$ & {$[135]$} \\
\hline Rat & $\begin{array}{l}\text { acetaldehyd } \\
\text { e }\end{array}$ & $\begin{array}{l}\text { Saffron/ 62.5, } 125 \text {, } \\
250 \mathrm{mg} / \mathrm{kg} / \text { p.o. }\end{array}$ & N.M. & $\begin{array}{l}\text { inhibition of long-term } \\
\text { potentiation (LTP) in the rat } \\
\text { dentate gyros=>inhibited }\end{array}$ & [136] \\
\hline Mouse & ethanol & $\begin{array}{l}\text { Crocin/ 50, 100, } 200 \\
\mathrm{mg} / \mathrm{kg} / \text { p.o. }\end{array}$ & PAT & $\downarrow$ No. of errors, $\uparrow$ STL & [137] \\
\hline
\end{tabular}

Table 2. Results based on in vitro assays.

\begin{tabular}{l|l|l|l|l}
$\begin{array}{l}\text { Targeted } \\
\text { Cell Lines }\end{array}$ & Model & $\begin{array}{l}\text { Targeted Compound/ Exposure } \\
\text { Time/ } \\
\text { Dosage }\end{array}$ & Major outcomes & Ref. \\
\hline PC12 & $\begin{array}{l}\text { Aluminum } \\
\text { maltolate }\end{array}$ & $\begin{array}{l}\text { Saffron/ } 48 \text { hours/ } 50 \text { and } 100 \\
\mu \mathrm{g} / \mathrm{ml}\end{array}$ & $\begin{array}{l}\uparrow c e l l \text { viability, } \downarrow \text { total apoptotic cells, } \\
\downarrow \text { CAT activity }\end{array}$ & {$[138]$}
\end{tabular}




\begin{tabular}{|c|c|c|c|c|}
\hline $\begin{array}{l}\text { Targeted } \\
\text { Cell Lines }\end{array}$ & Model & $\begin{array}{l}\text { Targeted Compound/ Exposure } \\
\text { Time/ } \\
\text { Dosage }\end{array}$ & Major outcomes & Ref. \\
\hline PC12 & $\begin{array}{l}\mathrm{A} \beta(25-35), \\
\mathrm{H} 2 \mathrm{O} 2\end{array}$ & $\begin{array}{l}\text { saffron extract, essential oil, } \\
\text { safranal/ } 120 \mathrm{~min} / \text { saffron extract, } \\
\text { essential oil, safranal }(2.5,5,10 \text {, } \\
20,40,80 \mu \mathrm{M})\end{array}$ & $\begin{array}{l}\uparrow \text { cell survival rates, } \downarrow \text { fluorescence } \\
\text { intensity, } \downarrow \text { ROS production, } \downarrow \text { amount } \\
\text { of apoptosis, } \downarrow \text { Cyt, and cleaved } \\
\text { caspase } 3 \text {, increased phosphor } \\
\text { SAPK/JNK to SAPK/JNK=>inverted, } \\
\text { reduction in surviving and the ratio of } \\
\text { Phospho-p44/42 MAPK (ERK1/2) to } \\
\text { p44/42 MAPK (ERK1/2) and increase } \\
\text { in Phospho-PI3 kinase p } 85 / \text { p55 to PI3 } \\
\text { kinase p85/p55=>protected }\end{array}$ & [139] \\
\hline HT22 & $\begin{array}{l}\text { L-glutamate } \\
\text { (L-Glu) }\end{array}$ & Crocin/ $3 \mathrm{~h} / 0.5$ and $2 \mu \mathrm{M}$ & $\begin{array}{l}\text { L-Glu-induced mitochondrial } \\
\text { apoptosis in HT22 cells against } \\
\text { =>protected, MMP } \\
\text { dissipation=>restored, hyper-levels of } \\
\text { intracellular ROS compared with the } \\
\text { L-Glu-damaged HT22 } \\
\text { cells=>suppressed, an overload of } \\
\text { Ca2+=>inhibited, Bcl-xL, Bax, Bad, } \\
\text { caspase-3=>restored to standard } \\
\text { levels, attenuation the phosphorylation } \\
\text { of Akt and mTOR=>reversed }\end{array}$ & [81] \\
\hline N.M. & N.M. & Crocin/ $50-800 \mu \mathrm{M}$ & AChE; IC 50 $=0.92 \pm 0.23$ & {$[140]$} \\
\hline $\begin{array}{l}\text { SH-SY5Y, } \\
\text { PC12 }\end{array}$ & $\begin{array}{l}\text { SH-SY5Y-APP, } \\
\text { PC12-htau }\end{array}$ & $\begin{array}{l}\text { trans-Crocin } 4 \text {, trans-Crocetin/ } 24, \\
72 \text { hours/ } 0.1,1,10,100,1000 \\
\mu \mathrm{M}\end{array}$ & $\begin{array}{l}\text { cell viability }=>\text { NSD, } \\
\text { Trans-crocin } 4 \text {; } \downarrow \text { total PSEN1, } \downarrow \text { ( } \gamma \text { - } \\
\text { secretases)PSEN1 and PSEN2 } \\
\text { complexes, } \uparrow \text { PSEN1-CTF and PSEN2, } \\
\downarrow \text { ( } \beta \text {-secretase)BACE1, sAPP } \downarrow \downarrow \text {, APP- } \\
\text { C83=>NSD, } \downarrow \text { APP-C99 } \\
\text { Trans-crocetin; total APP, } \uparrow \text { cellular } \\
\text { APP and APP-C99 } \\
\text { trans-crocin 4; total tau levels } \downarrow, \downarrow \text { tau } \\
\text { phosphorylation (pThr231 and } \\
\text { pSer199/Ser202-tau), active and } \\
\text { inactive forms of GSK3 } \beta \text { (total } \\
\text { GSK3 } \beta, \text { pSer9-GSK3 } \beta \text { ) and ERK1/2 } \\
\text { (total ERK2, pERK1, } \\
\text { pERK2)=>downregulated, } \\
\text { ERK1=>NSD, } \\
\text { trans-crocetin; } \downarrow \text { total and } \\
\text { phosphorylated tau (pThr231 and } \\
\text { pSer199/Ser202), as well as GSK3 } \beta, \\
\text { ERK2, pERK1 and pERK2, } \uparrow \text { inactive } \\
\text { pSer9-GSK3 } \beta\end{array}$ & [141] \\
\hline HeLa cells & APP751 & Crocetin/ $8 \mathrm{~h} / 10,20,40 \mu \mathrm{M}$ & $\begin{array}{l}\text { cell viability }=>\text { NSD, } \downarrow A \beta 42 \text { and } \\
A \beta 40\end{array}$ & [93] \\
\hline N.M. & N.M. & $\begin{array}{l}\text { saffron, crocin-1, crocin-2, } \\
\text { crocetin, and safranal), and } \\
\text { crocetin structural analogs/ } 0.233 \text {, } \\
0.669,2.33,6.99,23.3 \mu \mathrm{g} / \mathrm{mL}\end{array}$ & $\begin{array}{l}\text { Saffron; inhibited } \alpha \mathrm{S} \text { aggregation and } \\
\text { dissociated } \alpha \mathrm{S} \text { fibril; IC50=0.656 and } \\
\text { EC50=4.17 } \mu \mathrm{g} / \mathrm{mL} \text {, fibrils of } \alpha \mathrm{S} \downarrow \text {, } \\
\text { Crocin- } 1, \text { crocin-2, crocetin, and } \\
\text { norbixin inhibited } \alpha \mathrm{S} \text { aggregation and } \\
\text { dissociated } \alpha \mathrm{S} \text { fibrils, with IC50 } \\
\text { values of } 4.00,0.541,0.0930 \text { (first), } \\
\text { and } 0.911 \mu \mathrm{M}, \text { respectively, and EC50 } \\
\text { values of } 4.95,3.63,0.0617 \text { (first), } \\
\text { and } 0.244 \mu \mathrm{M} / \text { safranal, } \\
\text { hexadecanedioic acid, and trans, trans- } \\
\text { muconic acid; did not show }>50 \% \\
\text { inhibition and }>50 \% \text { efficacy }\end{array}$ & [142] \\
\hline $\begin{array}{l}\text { LS-180, SH- } \\
\text { SY5Y, and } \\
\text { THP-1 }\end{array}$ & $\begin{array}{l}\text { NLRP3 } \\
\text { inflammasom }\end{array}$ & IIIM-141/ $100 \mu \mathrm{g} / \mathrm{mL}$ & $\begin{array}{l}\text { IL-1 } \beta=>\text { inhibited, } \uparrow \text { efflux of the } \\
\text { Rh123 dye, } \downarrow \% \text { intracellular Rh123 } \\
\text { levels in LS- } 180 \text { cells, } \uparrow \text { protection of } \\
\text { SH-SY5Y cells from amyloid- } \beta \\
\text { toxicity, and protection of SH-SY5Y } \\
\text { cells from glutamate toxicity, } \\
\uparrow \text { expression of P-gp and the amyloid- } \beta \\
\text { clearance }\end{array}$ & [100] \\
\hline
\end{tabular}




\begin{tabular}{|c|c|c|c|c|}
\hline $\begin{array}{l}\text { Targeted } \\
\text { Cell Lines }\end{array}$ & Model & $\begin{array}{l}\text { Targeted Compound/ Exposure } \\
\text { Time/ } \\
\text { Dosage }\end{array}$ & Major outcomes & Ref. \\
\hline bEnd3 & $\begin{array}{l}\text { BBB } \\
\text { endothelium }\end{array}$ & Saffron $/ 1.1,2.2 \mu \mathrm{g} / \mathrm{mL}$ & $\begin{array}{l}\uparrow \text { tightness of bEnd- } 3 \text { cells-based BBB } \\
\text { model, } \uparrow \text { transport of } 125 \mathrm{I}-\mathrm{A} \beta 40 \text { from } \\
\text { basolateral to apical compartment } \\
\text { across the bEnd- } 3 \text { cells, } \uparrow \text { P-gp } \\
\text { expression, LRP1 expression=>NSD }\end{array}$ & [109] \\
\hline $\begin{array}{l}\text { AD patients' } \\
\text { monocytes }\end{array}$ & $\mathrm{A} \beta 1-42$ & $\begin{array}{l}\text { trans-crocetin/ } 24,48,72 \text {, and } 120 \\
\mathrm{~h} / 5,10,25,50,100,150 \mu \mathrm{M}\end{array}$ & $\begin{array}{l}\downarrow \text { cell death, } \uparrow \mathrm{A} \beta 42 \text { degradation, } \\
\uparrow \mathrm{A} \beta 42 \text { degradation through CatB }\end{array}$ & [143] \\
\hline EA.hy926 & $\mathrm{H} 2 \mathrm{O} 2$ & $\begin{array}{l}\text { Saffron in a combination } / 24 \mathrm{~h} / \\
8.25,16.5,33 \mathrm{mg} / \mathrm{kg}\end{array}$ & $\begin{array}{l}\downarrow \text { ROS-related cellular damage, } \downarrow \text { LDH } \\
\text { leakage } \uparrow \uparrow \text { SOD, Intracellular ROS } \\
\text { Generation=>suppressed, } \downarrow \text { Bax/Bcl-2 } \\
\text { ratio, } \downarrow \text { cleaved caspase-3 expression }\end{array}$ & [144] \\
\hline NSCs & $\mathrm{A} \beta 1-42$ & Saffron $/ 26 \mathrm{~h} / 25 \mu \mathrm{g} / \mathrm{ml}$ & $\begin{array}{l}\uparrow \text { nuclear intensities of caspase- } 3 \\
\text { activation in A } \beta \text {-treated samples, } \uparrow \\
\text { total No. of ROIs indicated expression } \\
\text { of c-fos concerning their nuclei }\end{array}$ & [145] \\
\hline SH-SY5Y & $\mathrm{A} \beta$ & $\begin{array}{l}\text { Crocin/ } 24 \mathrm{~h} \\
1,10,50 \mu \mathrm{M}\end{array}$ & $\begin{array}{l}\downarrow \text { fluorescence intensity, } \uparrow \text { cell viability, } \\
\text { intracellular ROS level=>inhibited, } \\
\text { formation of A } \beta \text { fibril=>inhibited }\end{array}$ & [146] \\
\hline N.M. & N.M. & Saffron/ 50, $250 \mu \mathrm{g} / \mathrm{mL}$ & $\beta$-secretase $=>$ inhibited & [147] \\
\hline N.M. & N.M. & $\begin{array}{l}\text { Saffron pollens in a combination } \\
\text { in alcoholic and nonalcoholic } \\
\text { beverage }\end{array}$ & $\begin{array}{l}\text { Nonalcoholic; DPPH=> } 91.95 \pm 0.23 \\
\text { (mg trolox/100 mL beverages) } \\
\text { FRAP }=>2.51 \pm 0.01(\text { mmol } \\
\text { Fe(II)/100 mL beverages) } \\
\text { Alcoholic; DPPH }=>66.19 \pm 0.17 \\
\text { FRAP }=>1.28 \pm 0.02 / \downarrow \text { AchE }\end{array}$ & [148] \\
\hline PC12 cells & $\begin{array}{l}1 \mathrm{~N} / 4 \mathrm{R} \text { human } \\
\text { tau protein }\end{array}$ & Crocin $/ 24 \mathrm{~h} / 100 \mu \mathrm{g} / \mathrm{mL}$ & tau aggregation=>inhibited & [149] \\
\hline HT22 cells & $\begin{array}{l}\mathrm{H} 2 \mathrm{O} 2, \\
\mathrm{~A} \beta 1-42\end{array}$ & Crocetin/ $24 \mathrm{~h} / 3 \mu \mathrm{M}$ & $\begin{array}{l}\text { HT22 cells H2O2-induced cell } \\
\text { death=>protected, HT22 cells A } \beta 1 \text { - } \\
42 \text {-induced cell death=> protected, } \\
\downarrow \text { ROS production induced by } A \beta 1-42\end{array}$ & {$[150]$} \\
\hline N.M. & $\mathrm{A} \beta 42$ & Crocin/ $15.4 \mu \mathrm{M}$ & $\begin{array}{l}\downarrow \text { fluorescence intensity (degree of } \\
\text { amyloid assembly), } \downarrow \text { ANS } \\
\text { fluorescence intensity (hydrophobic } \\
\text { surface exposure of A } \beta 42 \text { ), } \downarrow \text { number } \\
\text { of fibrils }\end{array}$ & [151] \\
\hline N.M. & $\mathrm{A} \beta 1-40$ & Crocin $/ 15 \mu \mathrm{g}$ & $\begin{array}{l}\downarrow \text { ThT fluorescence intensity, } \downarrow \text { ANS } \\
\text { fluorescence, } \downarrow \text { number, and average } \\
\text { fibril length }\end{array}$ & [152] \\
\hline N.M. & N.M. & Saffron & $\begin{array}{l}\downarrow \mathrm{AChE} / \mathrm{IC} 50 \text { of crocetin, } \\
\text { dimethylcrocetin, safranal=>96.33, } \\
107.1, \text { and } 21.09 \mu \mathrm{M} \text { respectively }\end{array}$ & [153] \\
\hline SH-SY5Y & $\mathrm{H} 2 \mathrm{O} 2$ & $\begin{array}{l}\text { Saffron; } 1,10,50125,250 \\
\mu \mathrm{g} / \mathrm{mL} / \text { Crocetin; 0.1, 1, 10, 50, } \\
125 \mu \mathrm{M} / \text { Safranal; 0.1, 1, 10, 50, } \\
125 \mu \mathrm{M} / 18 \mathrm{~h}\end{array}$ & $\begin{array}{l}\mathrm{H} 2 \mathrm{O} 2 \text {-induced toxicity on SH- } \\
\mathrm{SY} 5 \mathrm{Y} \text { (saffron, safranal, } \\
\text { crocetin)=>prevented, rescuing cell } \\
\text { viability, } \downarrow \text { ROS production, } \downarrow \text { H2O2- } \\
\text { induced caspase- } 3 \text { activation }\end{array}$ & [130] \\
\hline N.M. & $\mathrm{A} \beta$ & Crocetin & $\begin{array}{l}\text { ThT fluorescence }=>\text { NSD, A11- } \\
\text { positive conformational epitopes of } \\
\text { A } \beta \text { oligomers }=>\text { preserved }\end{array}$ & [154] \\
\hline N.M. & $\alpha$-Lactalbumin & Crocin, Safranal/ 50, $100 \mu \mathrm{M}$ & $\begin{array}{l}\downarrow \text { fluorescence intensity, } \downarrow \text { ANS } \\
\text { fluorescence intensity, } \uparrow \text { cell viability }\end{array}$ & [155] \\
\hline $\begin{array}{l}\text { BV2 } \\
\text { microglial } \\
\text { cells }\end{array}$ & $\begin{array}{l}\text { (LPS), A } \beta 25-35 \\
+ \text { IFN- } \gamma\end{array}$ & $\begin{array}{l}\text { Crocin, crocetin/ } 24 \mathrm{~h} / 5,10,20 \\
\mu \mathrm{M}\end{array}$ & $\begin{array}{l}\downarrow N O \text { production, } \uparrow \text { cell viability, } \\
\downarrow \text { TNF- } \alpha, \downarrow \text { IL- } 1 \beta, \downarrow \text { ROS production, } \\
\downarrow N F-\kappa B \text { activation, neuronal } \\
\text { damage }=>\text { protected }\end{array}$ & [156] \\
\hline N.M. & N.M. & saffron & $\mathrm{AChE}=>\mathrm{NSD}$ & [157] \\
\hline N.M. & $\mathrm{A} \beta 1-40$ & saffron, crocin & $\begin{array}{l}\text { antioxidant activity=>observed, } \\
\downarrow \text { fluorescence intensity }\end{array}$ & [158] \\
\hline PC-12 cells & TNF- $\alpha$ & Crocin/ $24 \mathrm{~h} / 1,10 \mu \mathrm{M}$ & $\begin{array}{l}\downarrow \text { expression of Bcl-2, morphological } \\
\text { changes and DNA } \\
\text { fragmentation=>blocked }\end{array}$ & [159] \\
\hline $\begin{array}{l}\text { hippocampal } \\
\text { slices }\end{array}$ & ethanol & Crocin/ $10 \mu \mathrm{M}$ & $\begin{array}{l}\text { inhibition of non-NMDA } \\
\text { response=>NSD, inhibition of NMDA } \\
\text { response=>significantly blocked }\end{array}$ & [160] \\
\hline
\end{tabular}




\begin{tabular}{l|l|l|l|l}
$\begin{array}{l}\text { Targeted } \\
\text { Cell Lines }\end{array}$ & Model & $\begin{array}{l}\text { Targeted Compound/ Exposure } \\
\text { Time/ } \\
\text { Dosage }\end{array}$ & Major outcomes & Ref. \\
\hline $\begin{array}{l}\text { hippocampal } \\
\text { slices }\end{array}$ & ethanol & Crocin/ $10,20,30 \mu \mathrm{M}$ & $\begin{array}{l}\uparrow \text { Spike amplitude, impairment of } \\
\text { hippocampal synaptic } \\
\text { plasticity=> prevented }\end{array}$ & {$[161]$}
\end{tabular}

Table 3. Results of clinical trials.

\begin{tabular}{|c|c|c|c|c|c|}
\hline Clinical Trial Type & $\begin{array}{l}\text { Patients' } \\
\text { Demographic } \\
\text { features }\end{array}$ & $\begin{array}{l}\text { Compound/ Route of } \\
\text { Administration/ } \\
\text { Duration } \\
\text { Dosage }\end{array}$ & test & Major outcomes & Ref. \\
\hline $\begin{array}{l}\text { Randomized Double- } \\
\text { Blind Placebo-Controlled } \\
\text { Trial }\end{array}$ & $\begin{array}{l}\text { Saffron; } N=18 \\
\text { Placebo; } N=19 \\
\text { Patients }<70 \\
\text { years and who } \\
\text { had aWMS-R } \\
\text { score }>70 \\
\end{array}$ & $\begin{array}{l}\text { saffron capsules/ p.o./ } \\
12 \text { weeks } \\
15 \text { mg/twice daily }\end{array}$ & $\begin{array}{l}\text { WMS-R, } \\
\text { HADS, } \\
\text { MMSE }\end{array}$ & NSD in scores & {$[162]$} \\
\hline $\begin{array}{l}\text { Randomized Double- } \\
\text { Blind Clinical Trial }\end{array}$ & $\begin{array}{l}\text { Herbal } \\
\text { combination; } \\
\mathrm{N}=30 \\
\text { Placebo; } \mathrm{N}=30, \\
\text { diagnosed } \mathrm{MCD} \\
\text { patients, mean } \\
\text { age of patients } \\
=66.8 \text { years, } \\
\text { mean age of } \\
\text { placebo }=70.2 \\
\text { years }\end{array}$ & $\begin{array}{l}\text { Saffron in a } \\
\text { combination/ p.o./ 4, } 8 \\
\text { weeks } \\
\text { (each capsule } \\
\text { containing } 500 \mathrm{mg} \text { of } \\
\text { sedge and } 30 \mathrm{mg} \text { of } \\
\text { saffron extract) plus a } \\
\text { teaspoon of Astragalus } \\
\text { honey (equal to } 5 \mathrm{~g} \text { in } \\
\text { each take)/ } 2 \text { capsules } \\
\text { per day }\end{array}$ & $\begin{array}{l}\text { ACE, } \\
\text { GDS }\end{array}$ & $\begin{array}{l}\text { Attention, } \\
\text { memory, } \\
\text { language, } \\
\text { and visuospatial } \\
\text { function=> } \\
\text { significant } \\
\text { changes, fluency } \\
\text { variable=>NSD, } \\
\text { MMSE, ACE, } \\
\text { GDS } \\
\text { scores=> significant } \\
\text { changes }\end{array}$ & [163] \\
\hline $\begin{array}{l}\text { Pilot, Double-Blind, } \\
\text { Randomized Clinical } \\
\text { Trial }\end{array}$ & $\begin{array}{l}30 \text { young elderly } \\
\text { subjects (mean } \\
\text { age } 66 \pm 3 \text { ), } \\
\text { MMSE=between } \\
20 \text { and } 27\end{array}$ & $\begin{array}{l}\text { Saffron in a } \\
\text { combination/ p.o./ } 8 \\
\text { weeks/ Combined } \\
\text { nutraceutical }\end{array}$ & $\begin{array}{l}\text { MMSE, } \\
\text { PSQ } \\
\text { Index, } \\
\text { SRDS }\end{array}$ & $\begin{array}{l}\text { MMSE }=>\text { NSD, } \\
\downarrow \text { PSQ Index, } \\
\downarrow \text { SRDS }\end{array}$ & {$[164]$} \\
\hline $\begin{array}{l}\text { Single-Blind } \\
\text { Randomized, with } \\
\text { Parallel Groups, Clinical } \\
\text { Trial }\end{array}$ & $\begin{array}{l}\text { patient; } \mathrm{N}=17 \\
\text { control; } \mathrm{N}=18 \\
\text { mean age of } \\
\text { patients with } \\
\mathrm{MDI}=71.47 \\
\text { mean age of } \\
\text { control= } 69.72\end{array}$ & Saffron/ 12 months & $\begin{array}{l}\text { GDS, } \\
\text { FRSSD, } \\
\text { MMSE, } \\
\text { NPI, } \\
\text { MOCA } \\
\text { MRI, } \\
\text { EEG, and } \\
\text { ERP }\end{array}$ & $\begin{array}{l}\text { MMSE=>NSD, } \\
\downarrow \text { FRSSD, } \\
\text { MoCA=>NSD, } \\
\uparrow N P I, \\
\text { GDS=>NSD, MRI, } \\
\text { EEG, and ERP=> } \\
\text { showed } \\
\text { improvement in } \\
\text { specific domains }\end{array}$ & [165] \\
\hline $\begin{array}{l}\text { Phase } 2 \text { Clinical Trial, } \\
\text { Randomized, Multicenter, } \\
\text { Double-Blind, Placebo- } \\
\text { Controlled Study }\end{array}$ & $\begin{array}{l}\text { male and female } \\
\text { patients, } 40 \text { years } \\
\text { of age or older, } \\
\text { with a diagnosis } \\
\text { of probable mild } \\
\text { to moderate } \mathrm{VaD}\end{array}$ & $\begin{array}{l}\text { Saffron in a } \\
\text { combination/ } 26 \text { weeks }\end{array}$ & $\begin{array}{l}\text { V-ADAS- } \\
\text { cog, } \\
\text { ADCS- } \\
\text { CGIC), } \\
\text { MMSE, } \\
\text { CDR, } \\
\text { ADCS- } \\
\text { ADL, } \\
\text { CLOX, } \\
\text { EXIT25, } \\
\text { NPI, MRI } \\
\text { or CT }\end{array}$ & $\begin{array}{l}\text { The results of the } \\
\text { study will provide } \\
\text { high-quality } \\
\text { evidence on the } \\
\text { effect of SLT } \\
\text { capsule in patients } \\
\text { with } \mathrm{VaD} \text { and have } \\
\text { the potential to } \\
\text { establish a novel } \\
\text { therapeutic } \\
\text { approach for } \mathrm{VaD}\end{array}$ & [166] \\
\hline $\begin{array}{l}\text { Double-Blind } \\
\text { Randomized Clinical } \\
\text { Trial }\end{array}$ & $\begin{array}{l}\text { Male and female } \\
\text { outpatients older } \\
\text { than } 60 \text { years, } \\
\text { MMSE score of } \\
8-14 \text { at baseline } \\
\text { Saffron; N=34 } \\
\text { Memantine; } \\
\mathrm{N}=34\end{array}$ & $\begin{array}{l}\text { Saffron/ p.o./ } 12 \\
\text { months/ } 30 \mathrm{mg} / \text { day }\end{array}$ & $\begin{array}{l}\text { SCIRS, } \\
\text { FAST, } \\
\text { MMSE }\end{array}$ & $\begin{array}{l}\text { Scores=>NSD } \\
\text { between } \\
\text { memantine and } \\
\text { saffron groups }\end{array}$ & [167] \\
\hline $\begin{array}{l}\text { multicenter, randomized, } \\
\text { double-blind controlled } \\
\text { trial }\end{array}$ & $\begin{array}{l}\text { MMSE; } 15-26 \\
\text { inclusive } \\
54 \text { Persian- } \\
\text { speaking adults }\end{array}$ & $\begin{array}{l}\text { Saffron / p.o./ } 15 \mathrm{mg} \\
\text { for } 4 \text { weeks/ } 22 \text { weeks }\end{array}$ & $\begin{array}{l}\text { ADAS- } \\
\text { cog, CDR- } \\
\text { SB }\end{array}$ & Scores $=>$ NSD & [168] \\
\hline
\end{tabular}




\begin{tabular}{l|l|l|l|l|} 
& $\begin{array}{l}55 \text { years of age or } \\
\text { older }\end{array}$ & & \\
\hline $\begin{array}{l}\text { Randomized and Placebo- } \\
\text { Controlled Trial }\end{array}$ & $\begin{array}{l}56 \text { patients older } \\
\text { than 55 years } \\
\text { MMSE) score of } \\
15-26\end{array}$ & $\begin{array}{l}\text { Saffron capsules/ p.o./ } \\
16 \text {-week/ 15 mg twice } \\
\text { per day }\end{array}$ & $\begin{array}{l}\text { ADAS- } \\
\text { cog, CDR- } \\
\text { SB }\end{array}$ & $\begin{array}{l}\text { ADAS-cog, CDR- } \\
\text { SB=>significant } \\
\text { changes }\end{array}$
\end{tabular}

\section{Pharmacological and Pharmaceutical Aspects of Saffron}

From the collected results, it is concluded that saffron and its active constituents may be a great therapeutic agent combat cognitive dysfunctions. Toxic and lethal effects of saffron can be revealed in $5 \mathrm{~g}$ and $20 \mathrm{~g}$ of the daily dose, respectively. Therapeutic activities of saffron have been observed in (30-50mg/day) clinically; thus, saffron considered as a medicinal plant that possesses a wide therapeutic index (145)[170]. Clinical safety evaluation of crocin through a placebo-controlled, double-blind, randomized study has shown a safe and well-tolerated manifestation in healthy volunteers administered $20 \mathrm{mg} /$ day of crocin in one month [171].

Through studying the pharmacokinetic properties of saffron, it is indicated that oral administration of crocetin is a water-soluble carotenoid of saffron stigmas crocetin is absorbed and detected in plasma faster than the other carotenoids, through its excretion take place widely through the intestinal tract, plasma concentrations of crocetin are not accumulated following repeated doses crocin, hydrolysis of crocin is taken place mostly in the intestinal tract. Crocetin can penetrate the blood barrier brain (BBB) and reach to CNS; it has a high affinity to the NMDA receptor; thus, saffron applied in the treatment of CNS disorders $[172,173]$. The free form of crocetin may be bound to albumin in blood plasma, this weak bind cand break easily, and crocetin may be able to penetrate the tissues. To enhance the stability of crocin, chitosan, and alginate biopolymer carriers can be used for crocin delivery and encapsulation of bioactive agents $[29,174]$.

\section{Conclusions}

Various health complications can be treated by the application of medicinal plants, phytochemicals, and nutraceuticals. Discovery and identification of these components with possession of numerous clinical indications can ameliorate our lives quality. Saffron and its active phytochemicals comprising crocin, crocetin, and safranal have the capacity to elucidate anti-AD and memory enhancer properties. Mostly common contributed mechanisms to combat $\mathrm{AD}$ are underlying oxidative stress, inflammatory process, apoptosis, beta-amyloid aggregation, and tauopathy. Saffron can be used in the prevention and treatment of clinical complications with etiologies the same as AD. Moreover, regarding the capability of saffron to penetrate $\mathrm{BBB}$ it can be considered in therapeutic approaches of other neurological disorders. Study particular cell signaling pathways contributed in $\mathrm{AD}$ is recommended following saffron administration. From all of the mentioned extracted data of original researches, memoryenhancing properties of saffron are concluded. However, some modifications in carriers, bioavailability, and route of administration will be required.

\section{Funding}

The project financially supported by Shahid Beheshti University of Medical Sciences. 


\section{Acknowledgments}

The authors would like to appreciate Dr.H.Barabadi for his beneficial guidance.

\section{Conflicts of Interest}

The authors declare no conflict of interest.

\section{References}

1. Ibrahim, M.M.; Gabr, M.T. Multitarget therapeutic strategies for Alzheimer's disease. Neural Regen Res 2019, 14, 437-440, https://doi.org/10.4103/1673-5374.245463.

2. Shah, H.; Albanese, E.; Duggan, C.; Rudan, I.; Langa, K.M.; Carrillo, M.C.; Chan, K.Y.; Joanette, Y.; Prince, M.; Rossor, M.; Saxena, S.; Snyder, H.M.; Sperling, R.; Varghese, M.; Wang, H.; Wortmann, M.; Dua, T. Research priorities to reduce the global burden of dementia by 2025. Lancet Neurol 2016, 15, 1285-1294, https://doi.org/10.1016/s1474-4422(16)30235-6.

3. Noroozian, M. Alzheimer's Disease. Neurol Clin 2016, 34, 69-131, https://doi.org/10.1016/j.ncl.2015.08.005.

4. International, A.S.D. World Alzheimer Report 2019: Attitudes to dementia. London: Alzheimer's Disease International 2019.

5. Meraz-Ríos, M.A.; Toral-Rios, D.; Franco-Bocanegra, D.; Villeda-Hernández, J.; Campos-Peña, V. Inflammatory process in Alzheimer's Disease. Front Integr Neurosci 2013, 7, 59-59, https://doi.org/10.3389/fnint.2013.00059.

6. Roher, A.; Kokjohn, T.; Clarke, S.; Sierks, M.; Maarouf, C.; Serrano, G.; Sabbagh, M.; Beach, T. APP/A $\beta$ structural diversity and Alzheimer's disease pathogenesis. Neurochem Int 2017, 110, https://doi.org/10.1016/j.neuint.2017.08.007.

7. Du, S.; Readel, E.R.; Wey, M.; Armstrong, D.W. Complete identification of all 20 relevant epimeric peptides in $\beta$-amyloid: a new HPLC-MS based analytical strategy for Alzheimer's research. Chem Commun 2020, 56, 1537-1540, https://doi.org/10.1039/C9CC09080K.

8. Rajendran, L.; Honsho, M.; Zahn, T.R.; Keller, P.; Geiger, K.D.; Verkade, P.; Simons, K. Alzheimer's disease $\beta$-amyloid peptides are released in association with exosomes. Proc Natl Acad Sci 2006, 103, 11172 11177, https://doi.org/10.1073/pnas.0603838103.

9. Nandakumar, A.; Xing, Y.; Aranha, R.R.; Faridi, A.; Kakinen, A.; Javed, I.; Koppel, K.; Pilkington, E.H.; Purcell, A.W.; Davis, T.P.; Faridi, P.; Ding, F.; Ke, P.C. Human Plasma Protein Corona of A $\beta$ Amyloid and Its Impact on IAPP Cross-Seeding. Biomacromolecules 2020, 21, 988-998, https://doi.org/10.1021/acs.biomac.9b01650.

10. Karch, C.M.; Cruchaga, C.; Goate, A.M. Alzheimer's disease genetics: from the bench to the clinic. Neuron 2014, 83, 11-26, https://doi.org/10.1016/j.neuron.2014.05.041.

11. Oliver, D.; Reddy, P.H. Small molecules as therapeutic drugs for Alzheimer's disease. Mol Cell Neurosci 2019, 96, https://doi.org/10.1016/j.mcn.2019.03.001.

12. Näslund, J.; Haroutunian, V.; Mohs, R.; Davis, K.L.; Davies, P.; Greengard, P.; Buxbaum, J.D. Correlation Between Elevated Levels of Amyloid $\beta$-Peptide in the Brain and Cognitive Decline. JAMA 2000, 283, 15711577, https://doi.org/10.1001/jama.283.12.1571.

13. Aprahamian, I.; Stella, F.; Forlenza, O. New treatment strategies for Alzheimer's disease: Is there a hope? Indian J Med Res 2013, 138, 449-460.

14. Kozauer, N.; Katz, R. Regulatory innovation and drug development for early-stage Alzheimer's disease. $N$ Engl J Med 2013, 368, 1169-1171, https://doi.org/10.1056/nejmp1302513.

15. Vaisi-Raygani, A.; Rahimi, Z.; Zahraie, M.; Noroozian, M.; Pourmotabbed, A. Association between enzymatic and nonenzymatic antioxidant defense with Alzheimer disease. Acta Med Iran 2008, 46, 11-16.

16. Manayi, A.; Saeidnia, S.; Gohari, A.R.; Abdollahi, M. Methods for the discovery of new anti-aging products - targeted approaches. Expert Opin Drug Discov 2014, 9, 383-405, https://doi.org/10.1517/17460441.2014.885014.

17. Small, G.W.; Ercoli, L.M.; Silverman, D.H.; Huang, S.C.; Komo, S.; Bookheimer, S.Y.; Lavretsky, H.; Miller, K.; Siddarth, P.; Rasgon, N.L. Cerebral metabolic and cognitive decline in persons at genetic risk for Alzheimer's disease. Proc Natl Acad Sci 2000, 97, 6037-6042, https://doi.org/10.1073/pnas.090106797.

18. Farkhondeh, T.; Azimi-Nezhad, M.; Samini, F.; Pourbagher-Shahri, A.M.; Samarghandian, S. Neuroprotective effect of alpinia galanga against middle cerebral artery occlusion-induced ischemia in rat. Biointerface Res Appl Chem 2020, 10, 6273-6281. 
19. Rosini, M.; Simoni, E.; Minarini, A.; Melchiorre, C. Multi-target Design Strategies in the Context of Alzheimer's Disease: Acetylcholinesterase Inhibition and NMDA Receptor Antagonism as the Driving Forces. Neurochem Res 2014, 39, 1914-1923, https://doi.org/10.1007/s11064-014-1250-1.

20. Hampel, H. Current insights into the pathophysiology of Alzheimer's disease: selecting targets for early therapeutic intervention. Int Psychogeriatr 2012, 24, S10-S17, https://doi.org/10.1017/s1041610212000579.

21. Yazdani, E.; Talebi, M.; Zarshenas, M.; Moein, M. Evaluation of possible antioxidant activities of barberry solid formulation, a selected formulation from Traditional Persian Medicine (TPM) via various procedures. Biointerface Res Appl Chem 2019, 9, 4517-4521, https://doi.org/10.33263/BRIAC96.517521.

22. Talebi, M.; Zarshenas, M.M.; Moein, M.; Yazdani, E. Experimental evidence of the antioxidant effect of a multi-ingredient traditional Persian formulation with hepatoprotective activity. In: Proceedings of 12th International Congress Laboratory and Clinical Sciences, Tehran, Iran; pp. 931-932.

23. Howes, M.J.R.; Perry, E. The Role of Phytochemicals in the Treatment and Prevention of Dementia. Drugs Aging 2011, 28, 439-468, https://doi.org/10.2165/11591310-000000000-00000.

24. Russo, P.; Frustaci, A.; Bufalo, A.D.; Fini, M.; Cesario, A. Multitarget drugs of plants origin acting on alzheimer's disease. Curr Med Chem 2013, 20, 1686-1693, https://doi.org/10.2174/0929867311320130008.

25. Alavizadeh, S.H.; Hosseinzadeh, H. Bioactivity assessment and toxicity of crocin: a comprehensive review. Food Chem Toxicol 2014, 64, 65-80, https://doi.org/10.1016/j.fct.2013.11.016.

26. Akhondzadeh, S. Crocus sativus (saffron), an herb with a history as long as the history of iran with psychotropic effects. J Med Plants 2016, 15, 1-6.

27. Farkhondeh, T.; Azimi-Nezhad, M.; Samini, F.; Pourbagher-Shahri, A.M.; Samarghandian, S. Neuroprotective effect of alpinia galanga against middle cerebral artery occlusion-induced ischemia in rat. Biointerface Res Appl Chem 2020, 10, 6273-6281.

28. Rezaee, R.; Hosseinzadeh, H. Safranal: from an aromatic natural product to a rewarding pharmacological agent. Iran J Basic Med Sci 2013, 16, 12-26.

29. Rahaiee, S.; Hashemi, M.; Shojaosadati, S.A.; Moini, S.; Razavi, S.H. Nanoparticles based on crocin loaded chitosan-alginate biopolymers: Antioxidant activities, bioavailability and anticancer properties. Int J Biol Macromol 2017, 99, 401-408, https://doi.org/10.1016/j.ijbiomac.2017.02.095.

30. Gohari, A.; Saeidnia, S.; kurepaz mahmoodabadi, M. An overview on saffron, phytochemicals, and medicinal properties. Pharmacogn Rev 2013, 7, 61-66, https://doi.org/10.4103/0973-7847.112850.

31. Talebi, M.; Patil, R.; Sidisky, L.; Berthod, A.; Armstrong, D. Branched-chain dicationic ionic liquids for fatty acid methyl ester assessment by gas chromatography. Anal Bioanal Chem 2017, 410, 1-11, https://doi.org/10.1007/s00216-017-0722-y.

32. Samarghandian, S.; Borji, A. Anticarcinogenic effect of saffron (Crocus sativus L.) and its ingredients. Pharmacognosy Res 2014, 6, 99-107, https://doi.org/10.4103/0974-8490.128963.

33. Bhandari, P.R. Crocus sativus L.(saffron) for cancer chemoprevention: a mini review. J Tradit Complement Med 2015, 5, 81-87, https://doi.org/10.1016/j.jtcme.2014.10.009.

34. Soeda, S.; Aritake, K.; Urade, Y.; Sato, H.; Shoyama, Y. Neuroprotective Activities of Saffron and Crocin. Adv Neurobiol 2016, 12, 275-292, https://doi.org/10.1007/978-3-319-28383-8_14.

35. Yazdi, H.; Samarghandian, S.; Farkhondeh, T. Effects of Crocins in the Management of Neurodegenerative Pathologies: A Review. Neurophysiology 2018, 50, https://doi.org/10.1007/s11062-018-9752-0.

36. Pitsikas, N. The effect of Crocus sativus L. and its constituents on memory: basic studies and clinical applications. Evid Based Complement Alternat Med 2015, 2015, https://doi.org/10.1155/2015/926284.

37. Farkhondeh, T.; Samarghandian, S.; Yazdi, H.; Samini, F. The protective effects of crocin in the management of neurodegenerative diseases: a review. Am J Neurodegener Dis 2018, 7, 1-10.

38. Hosseinzadeh, H. Saffron: A Herbal Medicine of Third Millennium. Jundishapur J Nat Pharm Prod 2014, 9, 1-2.

39. Baba, S.A.; Malik, A.H.; Wani, Z.A.; Mohiuddin, T.; Shah, Z.; Abbas, N.; Ashraf, N. Phytochemical analysis and antioxidant activity of different tissue types of Crocus sativus and oxidative stress alleviating potential of saffron extract in plants, bacteria, and yeast. $S$ Afr $J$ Bot. 2015, 99, 80-87, https://doi.org/10.1016/j.sajb.2015.03.194.

40. Farkhondeh, T.; Samarghandian, S. The effect of saffron (Crocus sativus L.) and its ingredients on the management of diabetes mellitus and dislipidemia. Afr J Pharm Pharmacol 2014, 8, 541-549, https://doi.org/10.5897/AJPPX2013.0006.

41. Rahmani, A.; Khan, A.; Aldebasi, Y. Saffron (Crocus sativus) and its Active Ingredients: Role in the Prevention and Treatment of Disease. Pharmacognosy J 2017, 9, 873-879, https://doi.org/10.5530/pj.2017.6.137.

42. Kamalipour, M.; Akhondzadeh, S. Cardiovascular effects of saffron: an evidence-based review. J Tehran Heart Cent 2011, 6, 59-61.

43. Khorasany, A.R.; Hosseinzadeh, H. Therapeutic effects of saffron (Crocus sativus L.) in digestive disorders: a review. Iran J Basic Med Sci 2016, 19, 455-469.

44. Hosseinzadeh, H.; Younesi, H.M. Antinociceptive and anti-inflammatory effects of Crocus sativus L. stigma and petal extracts in mice. BMC Pharmacol 2002, 2, https://dx.doi.org/10.1186\%2F1471-2210-2-7. 
45. Khazdair, M.R.; Boskabady, M.H.; Hosseini, M.; Rezaee, R.; Tsatsakis, A.M. The effects of Crocus sativus (saffron) and its constituents on nervous system: A review. Avicenna J Phytomed 2015, 5, 376-391.

46. Kyriakoudi, A.; Ordoudi, S.; Roldán-Medina, M.; Tsimidou, M. Saffron, a functional spice. Austin J Nutri Food Sci 2015, 3.

47. Shahi, T.; Assadpour, E.; Jafari, S.M. Main chemical compounds and pharmacological activities of stigmas and tepals of 'red gold'; saffron. Trends Food Sci Technol 2016, 58, 69-78, https://doi.org/10.1016/j.tifs.2016.10.010.

48. Bhargava, V. Medicinal uses and pharmacological properties of Crocus sativus Linn (Saffron). Int. J. Pharm. Pharm. Sci 2011, 3, 22-26.

49. Liberati, A.; Altman, D.G.; Tetzlaff, J.; Mulrow, C.; Gøtzsche, P.C.; Ioannidis, J.P.A.; Clarke, M.; Devereaux, P.J.; Kleijnen, J.; Moher, D. The PRISMA statement for reporting systematic reviews and metaanalyses of studies that evaluate healthcare interventions: explanation and elaboration. BMJ 2009, 339, b2700, https://doi.org/10.1136/bmj.b2700.

50. Hölter, S.; Garrett, L.; Einicke, J.; Sperling, B.; Dirscherl, P.; Zimprich, A.; Fuchs, H.; Gailus-Durner, V.; Angelis, M.; Wurst, W. Assessing Cognition in Mice. Curr Protoc Mouse Biol 2015, 5, 331-358, https://doi.org/10.1002/9780470942390.mo150068.

51. Farkhondeh, T.; Samarghandian, S; Sedaghat, M. Agents-induced nephrotoxicity and catcheins. Biointerface Res Appl Chem 2020, 10, 5028-5031.

52. Yu, T.; Guo, M.; Garza, J.; Rendon, S.; Sun, X.L.; Zhang, W.; Lu, X.Y. Cognitive and neural correlates of depression-like behaviour in socially defeated mice: an animal model of depression with cognitive dysfunction. Int J Neuropsychopharmacol 2011, 14, 303-317, https://doi.org/10.1017/S1461145710000945.

53. Albani, S.H.; Andrawis, M.M.; Abella, R.J.H.; Fulghum, J.T.; Vafamand, N.; Dumas, T.C. Behavior in the elevated plus maze is differentially affected by testing conditions in rats under and over three weeks of age. Front Behav Neurosci 2015, 9, 31-31, https://doi.org/10.3389/fnbeh.2015.00031.

54. Pocernich, C.B.; Butterfield, D.A. Elevation of glutathione as a therapeutic strategy in Alzheimer disease. Biochim Biophys Acta Mol Basis Dis 2012, 1822, 625-630, https://doi.org/10.1016/j.bbadis.2011.10.003.

55. Young, A.; Oldford, C.; Mailloux, R.J. Lactate dehydrogenase supports lactate oxidation in mitochondria isolated from different mouse tissues. Redox Biol 2020, 28, 101339-101339, https://doi.org/10.1016/j.redox.2019.101339.

56. Greilberger, J.; Fuchs, D.; Leblhuber, F.; Greilberger, M.; Wintersteiger, R.; Tafeit, E. Carbonyl Proteins as a Clinical Marker in Alzheimer's Disease and its Relation to Tryptophan Degradation and Immune Activation. Clin Lab 2010, 56, 441-448.

57. Kudo, W.; Lee, H.P.; Smith, M.A.; Zhu, X.; Matsuyama, S.; Lee, H.g. Inhibition of Bax protects neuronal cells from oligomeric A $\beta$ neurotoxicity. Cell Death Dis 2012, 3, e309-e309, https://doi.org/10.1038/cddis.2012.43.

58. Walker, D.; Lue, L.F. Anti-inflammatory and immune therapy for Alzheimer's disease: current status and future directions. Curr Neuropharmacol 2007, 5, 232-243, https://doi.org/157015907782793667.

59. Munoz, L.; Ammit, A.J. Targeting p38 MAPK pathway for the treatment of Alzheimer's disease. Neuropharmacology 2010, 58, 561-568, https://doi.org/10.1016/j.neuropharm.2009.11.010.

60. Song, J.; Kim, O.Y. Perspectives in Lipocalin-2: Emerging Biomarker for Medical Diagnosis and Prognosis for Alzheimer's Disease. Clin Nutr Res 2018, 7, 1-10, https://doi.org/10.7762/cnr.2018.7.1.1.

61. Casas, C. GRP78 at the Centre of the Stage in Cancer and Neuroprotection. Front Neurosci 2017, 11, https://doi.org/10.3389/fnins.2017.00177.

62. Jones, S.V.; Kounatidis, I. Nuclear Factor-Kappa B and Alzheimer Disease, Unifying Genetic and Environmental Risk Factors from Cell to Humans. Front Immunol 2017, 8, 1805-1805, https://doi.org/10.3389/fimmu.2017.01805.

63. Cunha, C.; Brambilla, R.; Thomas, K.L. A simple role for BDNF in learning and memory? Front Mol Neurosci 2010, 3, 1-1, https://doi.org/10.3389/neuro.02.001.2010.

64. Ortega-Martínez, S. A new perspective on the role of the CREB family of transcription factors in memory consolidation via adult hippocampal neurogenesis. Front Mol Neurosci 2015, 8, https://doi.org/10.3389/fnmol.2015.00046.

65. Maia, M.A.; Sousa, E. BACE-1 and $\gamma$-Secretase as Therapeutic Targets for Alzheimer's Disease. Pharmaceuticals (Basel) 2019, 12, 41, https://doi.org/10.3390/ph12010041.

66. Reichenbach, N.; Delekate, A.; Plescher, M.; Schmitt, F.; Krauss, S.; Blank, N.; Halle, A.; Petzold, G.C. Inhibition of Stat3-mediated astrogliosis ameliorates pathology in an Alzheimer's disease model. EMBO Mol Med 2019, 11, https://doi.org/10.15252/emmm.201809665.

67. Ceyzériat, K.; Ben Haim, L.; Denizot, A.; Pommier, D.; Matos, M.; Guillemaud, O.; Palomares, M.-A.; Abjean, L.; Petit, F.; Gipchtein, P.; Gaillard, M.-C.; Guillermier, M.; Bernier, S.; Gaudin, M.; Aurégan, G.; Joséphine, C.; Déchamps, N.; Veran, J.; Langlais, V.; Cambon, K.; Bemelmans, A.P.; Baijer, J.; Bonvento, G.; Dhenain, M.; Deleuze, J.-F.; Oliet, S.H.R.; Brouillet, E.; Hantraye, P.; Carrillo-de Sauvage, M.-A.; Olaso, R.; Panatier, A.; Escartin, C. Modulation of astrocyte reactivity improves functional deficits in mouse models of Alzheimer's disease. Acta Neuropathol Commun 2018, 6, 104, https://doi.org/10.1186/s40478018-0606-1. 
68. Tu, S.; Okamoto, S.I.; Lipton, S.A.; Xu, H. Oligomeric A $\beta$-induced synaptic dysfunction in Alzheimer's disease. Mol Neurodegener 2014, 9, https://doi.org/10.1186/1750-1326-9-48.

69. Rohn, T.T.; Head, E. Caspases as therapeutic targets in Alzheimer's disease: is it time to "cut" to the chase? Int J Clin Exp Pathol 2009, 2, 108-118.

70. Verite, J.; Janet, T.; Julian, A.; Chassaing, D.; Page, G.; Paccalin, M. Peripheral blood mononuclear cells of Alzheimer's disease patients control CCL4 and CXCL10 levels in a human blood brain barrier model. Curr Alzheimer Res 2017, 14, 1215-1228, https://doi.org/10.2174/1567205014666170417110337.

71. Copanaki, E.; Schürmann, T.; Eckert, A.; Leuner, K.; Müller, W.E.; Prehn, J.H.M.; Kögel, D. The amyloid precursor protein potentiates CHOP induction and cell death in response to ER Ca2+ depletion. Biochim Biophys Acta Mol Cell Res 2007, 1773, 157-165, https://doi.org/10.1016/j.bbamcr.2006.10.002.

72. Stuart, K.E.; King, A.E.; Fernandez-Martos, C.M.; Summers, M.J.; Vickers, J.C. Environmental novelty exacerbates stress hormones and A $\beta$ pathology in an Alzheimer's model. Sci Rep 2017, 7, 2764-2764, https://doi.org/10.1038/s41598-017-03016-0.

73. Wang, X.X.; Tan, M.S.; Yu, J.T.; Tan, L. Matrix metalloproteinases and their multiple roles in Alzheimer's disease. Biomed Res Int 2014, 2014, 908636-908636, https://doi.org/10.1155/2014/908636.

74. Zamora-Martinez, E.R.; Edwards, S. Neuronal extracellular signal-regulated kinase (ERK) activity as marker and mediator of alcohol and opioid dependence. Front Integr Neurosci 2014, 8, 24-24, https://doi.org/10.3389/fnint.2014.00024.

75. Ostrowski, P.P.; Barszczyk, A.; Forstenpointner, J.; Zheng, W.; Feng, Z.P. Meta-Analysis of Serum InsulinLike Growth Factor 1 in Alzheimer's Disease. PLoS One 2016, 11, e0155733-e0155733, https://doi.org/10.1371/journal.pone.0155733.

76. Chang, J.R.; Ghafouri, M.; Mukerjee, R.; Bagashev, A.; Chabrashvili, T.; Sawaya, B.E. Role of p53 in neurodegenerative diseases. Neurodegener Dis 2012, 9, 68-80, https://doi.org/10.1159/000329999.

77. Mohammadzadeh, L.; Abnous, K.; Razavi, B.M.; Hosseinzadeh, H. Crocin-protected malathion-induced spatial memory deficits by inhibiting TAU protein hyperphosphorylation and antiapoptotic effects. Nutr Neurosci 2020, 23, 221-236, https://doi.org/10.1080/1028415x.2018.1492772.

78. Oddo, S. The role of mTOR signaling in Alzheimer disease. Front Biosci (Schol Ed) 2012, 4, 941-952, https://doi.org/10.2741/s310.

79. Karkoula, E.; Dagla, I.V.; Baira, E.; Kokras, N.; Dalla, C.; Skaltsounis, A.L.; Gikas, E.; Tsarbopoulos, A. A novel UHPLC-HRMS-based metabolomics strategy enables the discovery of potential neuroactive metabolites in mice plasma, following i.p. administration of the main Crocus sativus L. bioactive component. J Pharm Biomed Anal 2020, 177, https://doi.org/10.1016/j.jpba.2019.112878.

80. Zhang, Y.; Liu, J.; Yao, M.; Song, W.; Zheng, Y.; Xu, L.; Sun, M.; Yang, B.; Bensoussan, A.; Chang, D.; Li, H. Sailuotong Capsule Prevents the Cerebral Ischaemia-Induced Neuroinflammation and Impairment of Recognition Memory through Inhibition of LCN2 Expression. Oxid Med Cell Longev 2019, 2019, https://doi.org/10.1155/2019/8416105.

81. Wang, C.; Cai, X.; Hu, W.; Li, Z.; Kong, F.; Chen, X.; Wang, D. Investigation of the neuroprotective effects of crocin via antioxidant activities in HT22 cells and in mice with Alzheimer's disease. Int J Mol Med 2019, 43, 956-966, https://doi.org/10.3892/ijmm.2018.4032.

82. Shahidani, S.; Rajaei, Z.; Alaei, H. Pretreatment with crocin along with treadmill exercise ameliorates motor and memory deficits in hemiparkinsonian rats by anti-inflammatory and antioxidant mechanisms. Metab Brain Dis 2019, 34, 459-468, https://doi.org/10.1007/s11011-018-0379-z.

83. Sadoughi, D. The effect of crocin on apoptotic, inflammatory, BDNF, Pt, and A $\beta 40$ indicators and neuronal density of CA1, CA2, and CA3 regions of hippocampus in the model of Alzheimer suffering rats induced with trimethyltin chloride. Comp Clin Path 2019, 28, 1403-1413, https://doi.org/10.1007/s00580-01902981-4.

84. Negarandeh, Z.; Salamat, K.M.; Ali Hosseini, S.; Etemad, Z. The effect of endurance training with crocin consumption on IGF-1 and glycogen expression in rat hippocampus tissue of trimethyltin-treated model of Alzheimer's disease. Asian J Sports Med 2019, 10, https://doi.org/10.5812/asjsm.92246.

85. Mozaffari, S.; Ramezany Yasuj, S.; Motaghinejad, M.; Motevalian, M.; Kheiri, R. Crocin Acting as a Neuroprotective Agent against Methamphetamine-induced Neurodegeneration via CREB-BDNF Signaling Pathway. Iran J Pharm Res: IJPR 2019, 18, 745-758, https://doi.org/10.22037/ijpr.2019.2393.

86. Lin, L.; Liu, G.L.; Yang, L.N. Crocin Improves Cognitive Behavior in Rats with Alzheimer's Disease by Regulating Endoplasmic Reticulum Stress and Apoptosis. Biomed Res Int 2019, 2019, https://doi.org/10.1155/2019/9454913.

87. Ebrahimzadeh, A.; Moghadam, S.Y.; Rahimi, H.; Motaghinejad, M.; Motevalian, M.; Safari, S.; Mesrabadi, M.A. Crocin acts as a neuroprotective mediator against methylphenidate-induced neurobehavioral and neurochemical sequelae: Possible role of the CREB-BDNF signaling pathway. Acta Neurobiol Exp 2019, 79, 352-366.

88. Baluchnejadmojarad, T.; Mohamadi-Zarch, S.M.; Roghani, M. Safranal, an active ingredient of saffron, attenuates cognitive deficits in amyloid $\beta$-induced rat model of Alzheimer's disease: underlying mechanisms. Metab Brain Dis 2019, 34, 1747-1759, https://doi.org/10.1007/s11011-019-00481-6. 
89. Azarian, F.; Farsi, S.; Hosseini, S.A.; Azarbayjani, M.A. The effect of endurance training and crocin consumption on anxiety-like behaviors and aerobic power in rats with alzheimer's. Iran J Psychiatry Behav Sci 2019, 13, https://doi.org/10.5812/ijpbs.89011.

90. Asalgoo, S.; Jahromi, G.P.; Hatef, B.; Sahraei, H. The effect of saffron aqueous extract and crocin on PTSD rat models: The focus on learning and spatial memory. Journal of Zanjan University of Medical Sciences and Health Services 2019, 26, 34-42.

91. Adabizadeh, M.; Mehri, S.; Rajabpour, M.; Abnous, K.; Rashedinia, M.; Hosseinzadeh, H. The effects of crocin on spatial memory impairment induced by hyoscine: Role of NMDA, AMPA, ERK and CaMKII proteins in rat hippocampus. Iran $J$ Basic Med Sci 2019, 22, 601-609, https://doi.org/10.22038/ijbms.2019.30138.7266.

92. Zhang, X.Y.; Zhang, X.J.; Xv, J.; Jia, W.; Pu, X.Y.; Wang, H.Y.; Liang, H.; Zhuoma, L.; Lu, D.X. Crocin attenuates acute hypobaric hypoxia-induced cognitive deficits of rats. Eur J Pharmacol 2018, 818, 300-305, https://doi.org/10.1016/j.ejphar.2017.10.042.

93. Zhang, J.; Wang, Y.; Dong, X.; Liu, J. Crocetin attenuates inflammation and amyloid- $\beta$ accumulation in APPsw transgenic mice. Immun Ageing 2018, 15, https://doi.org/10.1186/s12979-018-0132-9.

94. Rezai, M.; Mahmoodi, M.; Kaeidi, A.; Karimabad, M.N.; Khoshdel, A.; Hajizadeh, M.R. Effect of crocin carotenoid on BDNF and CREB gene expression in brain ventral tegmental area of morphine treated rats. Asian Pac J Trop Biomed 2018, 8, 387-393, https://doi.org/10.4103/2221-1691.239426.

95. Pitsikas, N.; Tarantilis, P.A. Effects of the active constituents of Crocus sativus L. crocins and their combination with memantine on recognition memory in rats. Behav Pharmacol 2018, 29, 400-412, https://doi.org/10.1097/FBP.0000000000000380.

96. Khani, F.; Radahmadi, M.; Alaei, H.; Jafari, E. Effects of crocin on cognitive and spatial memories in rats under chronic isolation stress. Physiol Pharmacol (Iran) 2018, 22, 254-268.

97. Hadipour, M.; Kaka, G.; Bahrami, F.; Meftahi, G.H.; Jahromi, G.P.; Mohammadi, A.; Sahraei, H. Crocin improved amyloid beta induced long-term potentiation and memory deficits in the hippocampal CA1 neurons in freely moving rats. Synapse 2018, 72, https://doi.org/10.1002/syn.22026.

98. Delkhosh-Kasmaie, F.; Farshid, A.A.; Tamaddonfard, E.; Imani, M. The effects of safranal, a constitute of saffron, and metformin on spatial learning and memory impairments in type-1 diabetic rats: behavioral and hippocampal histopathological and biochemical evaluations. Biomed Pharmacother 2018, 107, 203-211, https://doi.org/10.1016/j.biopha.2018.07.165.

99. Dastgerdi, H.H.; Radahmadi, M.; Reisi, P.; Dastgerdi, A.H. Effect of Crocin, Exercise, and Crocinaccompanied Exercise on Learning and Memory in Rats under Chronic Unpredictable Stress. Adv Biomed Res 2018, 7, 137, https://doi.org/10.4103/abr.abr_153_18.

100. Bharate, S.S.; Kumar, V.; Singh, G.; Singh, A.; Gupta, M.; Singh, D.; Kumar, A.; Vishwakarma, R.A.; Bharate, S.B. Preclinical Development of Crocus sativus-Based Botanical Lead IIIM-141 for Alzheimer's Disease: Chemical Standardization, Efficacy, Formulation Development, Pharmacokinetics, and Safety Pharmacology. Acs Omega 2018, 3, 9572-9585, https://doi.org/10.1021/acsomega.8b00841.

101. Baghishani, F.; Mohammadipour, A.; Hosseinzadeh, H.; Hosseini, M.; Ebrahimzadeh-bideskan, A. The effects of tramadol administration on hippocampal cell apoptosis, learning and memory in adult rats and neuroprotective effects of crocin. Metab Brain Dis 2018, 33, 907-916, https://doi.org/10.1007/s11011-0180194-6.

102. Pitsikas, N.; Tarantilis, P.A. Crocins, the active constituents of Crocus sativus L., counteracted apomorphineinduced performance deficits in the novel object recognition task, but not novel object location task, in rats. Neurosci Lett 2017, 644, 37-42, https://doi.org/10.1016/j.neulet.2017.02.042.

103. Mazumder, A.G.; Sharma, P.; Patial, V.; Singh, D. Crocin Attenuates Kindling Development and Associated Cognitive Impairments in Mice via Inhibiting Reactive Oxygen Species-Mediated NF-B Activation. Basic Clin Pharmacol Toxicol 2017, 120, 426-433, https://doi.org/10.1111/bcpt.12694.

104. Linardaki, Z.I.; Lamari, F.N.; Margarity, M. Saffron (Crocus sativus L.) Tea Intake Prevents Learning/Memory Defects and Neurobiochemical Alterations Induced by Aflatoxin B1 Exposure in Adult Mice. Neurochem Res 2017, 42, 2743-2754, https://doi.org/10.1007/s11064-017-2283-Z.

105. Heidari, S.; Mehri, S.; Hosseinzadeh, H. Memory enhancement and protective effects of crocin against Dgalactose aging model in the hippocampus of wistar rats. Iran J Basic Med Sci 2017, 20, 1250-1259, https://doi.org/10.22038/IJBMS.2017.9541.

106. Dastgerdi, A.H.; Radahmadi, M.; Pourshanazari, A.A.; Dastgerdi, H.H. Effects of Crocin on Learning and Memory in Rats Under Chronic Restraint Stress with Special Focus on the Hippocampal and Frontal Cortex Corticosterone Levels. Adv Biomed Res 2017, 6, 157, https://doi.org/10.4103/abr.abr_107_17.

107. Cioancă, O.; Pagonakis, A.; Trifan, A.; Hrițcu, L.; Ioniţă, R.; Burlec, A.F.; Postu, P.; Cornelia, M.; Hăncianu, M. Pharmacognostic and pharmacologic screening of crocus sativus of Greek origin. Farmacia 2017, 65, 401-406.

108. Behravanfar, N.; Abnous, K.; Razavi, B.M.; Hosseinzadeh, H. Effects of crocin on spatial memory impairment induced by hyoscine and its effects on bdnf, creb, and p-creb protein and mrna levels in rat hippocampus. Jundishapur J Nat Pharm Prod 2017, 12, https://doi.org/10.5812/jjnpp.64315. 
109. Batarseh, Y.S.; Bharate, S.S.; Kumar, V.; Kumar, A.; Vishwakarma, R.A.; Bharate, S.B.; Kaddoumi, A. Crocus sativus Extract Tightens the Blood-Brain Barrier, Reduces Amyloid $\beta$ Load and Related Toxicity in 5XFAD Mice. ACS Chem Neurosci 2017, 8, 1756-1766, https://doi.org/10.1021/acschemneuro.7b00101.

110. Ahmadi, M.; Rajaei, Z.; Hadjzadeh, M.A.; Nemati, H.; Hosseini, M. Crocin improves spatial learning and memory deficits in the Morris water maze via attenuating cortical oxidative damage in diabetic rats. Neurosci Lett 2017, 642, 1-6, https://doi.org/10.1016/j.neulet.2017.01.049.

111. Yousefvand, N.; Doosti, H.; Pourmotabbed, A.; Nedaei, S.E. The therapeutic effect of crocin on ketamineinduced retrograde amnesia in rats. Journal of Kermanshah University of Medical Sciences 2016, 20, 68-73.

112. Rajaei, Z.; Hosseini, M.; Alaei, H. Effects of Crocin on brain oxidative damage and aversive memory in a 6-OHDA model of Parkinson's disease. Arq Neuropsiquiatr 2016, 74, 723-729, https://doi.org/10.1590/0004-282X20160131.

113. Samarghandian, S.; Azimi-Nezhad, M.; Samini, F. Preventive effect of safranal against oxidative damage in aged male rat brain. Exp Anim 2015, 64, 65-71, https://doi.org/10.1538/expanim.14-0027.

114. Rashedinia, M.; Lari, P.; Abnous, K.; Hosseinzadeh, H. Protective effect of crocin on acrolein-induced tau phosphorylation in the rat brain. Acta Neurobiol Exp 2015, 75, 208-219.

115. Ghaffari, S.; Hatami, H.; Dehghan, G. Saffron ethanolic extract attenuates oxidative stress, spatial learning, and memory impairments induced by local injection of ethidium bromide. Res Pharm Sci 2015, 10, $222-232$.

116. Asadi, F.; Jamshidi, A.H.; Khodagholi, F.; Yans, A.; Azimi, L.; Faizi, M.; Vali, L.; Abdollahi, M.; Ghahremani, M.H.; Sharifzadeh, M. Reversal effects of crocin on amyloid $\beta$-induced memory deficit: Modification of autophagy or apoptosis markers. Pharmacol Biochem Behav 2015, 139, 47-58, https://doi.org/10.1016/j.pbb.2015.10.011.

117. Samarghandian, S.; Azimi-Nezhad, M.; Samini, F. Ameliorative effect of saffron aqueous extract on hyperglycemia, hyperlipidemia, and oxidative stress on diabetic encephalopathy in streptozotocin induced experimental diabetes mellitus. Biomed Res Int 2014, 2014, https://doi.org/10.1155/2014/920857.

118. Naghizadeh, B.; Mansouri, M.T.; Ghorbanzadeh, B. Protective effects of crocin against streptozotocininduced oxidative damage in rat striatum. Acta Med Iran 2014, 52, 101-105.

119. Georgiadou, G.; Grivas, V.; Tarantilis, P.A.; Pitsikas, N. Crocins, the active constituents of Crocus Sativus L., counteracted ketamine-induced behavioural deficits in rats. Psychopharmacology 2014, 231, 717-726, https://doi.org/10.1007/s00213-013-3293-4.

120. Tashakori-Sabzevar, F.; Hosseinzadeh, H.; Motamedshariaty, V.S.; Movassaghi, A.R.; Mohajeri, S.A. Crocetin attenuates spatial learning dysfunction and hippocampal injury in a model of vascular dementia. Curr Neurovasc Res 2013, 10, 325-334, https://doi.org/10.2174/15672026113109990032.

121. Tamaddonfard, E.; Farshid, A.A.; Asri-Rezaee, S.; Javadi, S.; Khosravi, V.; Rahman, B.; Mirfakhraee, Z. Crocin improved learning and memory impairments in streptozotocin-induced diabetic rats. Iran J Basic Med Sci 2013, 16, 91-100.

122. Sadeghnia, H.R.; Kamkar, M.; Assadpour, E.; Boroushaki, M.T.; Ghorbani, A. Protective effect of safranal, a constituent of crocus sativus, on quinolinic acid-induced oxidative damage in rat hippocampus. Iran $J$ Basic Med Sci 2013, 16, 73-82.

123. Linardaki, Z.I.; Orkoula, M.G.; Kokkosis, A.G.; Lamari, F.N.; Margarity, M. Investigation of the neuroprotective action of saffron (Crocus sativus L.) in aluminum-exposed adult mice through behavioral and neurobiochemical assessment. Food Chem Toxicol 2013, 52, 163-170, https://doi.org/10.1016/j.fct.2012.11.016.

124. Naghibi, S.M.; Hosseini, M.; Khani, F.; Rahimi, M.; Vafaee, F.; Rakhshandeh, H.; Aghaie, A. Effect of aqueous extract of crocus sativus L. on morphine-induced memory impairment. Adv Pharmacol Sci 2012, 2012, https://doi.org/10.1155/2012/494367.

125. Khan, M.B.; Hoda, M.N.; Ishrat, T.; Ahmad, S.; Khan, M.M.; Ahmad, A.; Yusuf, S.; Islam, F. Neuroprotective efficacy of Nardostachys jatamansi and crocetin in conjunction with selenium in cognitive impairment. Neurol Sci 2012, 33, 1011-1020, https://doi.org/10.1007/s10072-011-0880-1.

126. Hosseinzadeh, H.; Sadeghnia, H.R.; Ghaeni, F.A.; Motamedshariaty, V.S.; Mohajeri, S.A. Effects of saffron (Crocus sativus L.) and its active constituent, crocin, on recognition and spatial memory after chronic cerebral hypoperfusion in rats. Phytother Res 2012, 26, 381-386, https://doi.org/10.1002/ptr.3566.

127. Dashti-r, M.H.; Zeinali, F.; Anvari, M.; Hosseini, S.M. Saffron (Crocus sativus L.) extract prevents and improves D-galactose and NaNO2 induced memory impairment in mice. EXCLI Journal 2012, 11, 328-337.

128. Cong, W.H.; Yang, B.; Xu, L.; Dong, X.X.; Sheng, L.S.; Hou, J.C.; Liu, J.X. Herbal Extracts Combination (WNK) Prevents Decline in Spatial Learning and Memory in APP/PS1 Mice through Improvement of Hippocampal A beta Plaque Formation, Histopathology, and Ultrastructure. Evid Based Complement Alternat Med 2012, https://doi.org/10.1155/2012/478190.

129. Shati, A.A.; Elsaid, F.G.; Hafez, E.E. Biochemical and molecular aspects of aluminium chloride-induced neurotoxicity in mice and the protective role of Crocus sativus L. extraction and honey syrup. Neuroscience 2011, 175, 66-74, https://doi.org/10.1016/j.neuroscience.2010.11.043.

130. Papandreou, M.A.; Tsachaki, M.; Efthimiopoulos, S.; Cordopatis, P.; Lamari, F.N.; Margarity, M. Memory enhancing effects of saffron in aged mice are correlated with antioxidant protection. Behav Brain Res 2011, 219, 197-204, https://doi.org/10.1016/j.bbr.2011.01.007. 
131. Ghadrdoost, B.; Vafaei, A.A.; Rashidy-Pour, A.; Hajisoltani, R.; Bandegi, A.R.; Motamedi, F.; Haghighi, S.; Sameni, H.R.; Pahlvan, S. Protective effects of saffron extract and its active constituent crocin against oxidative stress and spatial learning and memory deficits induced by chronic stress in rats. Eur J Pharmacol 2011, 667, 222-229, https://doi.org/10.1016/j.ejphar.2011.05.012.

132. Khalili, M.; Kiasalari, Z.; Rahmati, B.; Narenjkar, J. Behavioral and Histological Analysis of Crocus Sativus Effect in Intracerebroventricular Streptozotocin Model of Alzheimer Disease in Rats. Iran J Pathol $2010,5$.

133. Khalili, M.; Hamzeh, F. Effects of active constituents of crocus sativus L, crocin on streptozocin-induced model of sporadic Alzheimer's disease in male rats. Iran Biomed J 2010, 14, 59-65.

134. Khalili, M.; Roghani, M.; Ekhlasi, M. The effect of aqueous crocus sativus L. extract on intracerebroventricular streptozotocin-induced cognitive deficits in rat: a behavioral analysis. Iran J Pharm Res 2009, 8, 185-191.

135. Pitsikas, N.; Sakellaridis, N. Crocus sativus L. extracts antagonize memory impairments in different behavioural tasks in the rat. Behav Brain Res 2006, 173, 112-115, https://doi.org/10.1016/j.bbr.2006.06.005.

136. Abe, K.; Sugiura, M.; Yamaguchi, S.; Shoyama, Y.; Saito, H. Saffron extract prevents acetaldehyde-induced inhibition of long-term potentiation in the rat dentate gyrus in vivo. Brain Res 1999, 851, 287-289, https://doi.org/10.1016/S0006-8993(99)02174-5.

137. Sugiura, M.; Saito, H.; Nishiyama, N.; Shoyama, Y. Crocin Improves the Ethanol-induced Impairment of Learning Behaviors of Mice in Passive Avoidance Tasks. Proc Jpn Acad Ser B Phys Biol Sci 1995, 71, 319324, https://doi.org/10.2183/pjab.71.319.

138. Rahmani, S.; Saberzadeh, J.; Takhshid, M.A. The Hydroalcoholic Extract of Saffron Protects PC12 Cells against Aluminum-Induced Cell Death and Oxidative Stress in Vitro. Iran J Med Sci 2020, 45, 59-66, https://doi.org/10.30476/ijms.2019.44971.

139. Rafieipour, F.; Hadipour, E.; Emami, S.A.; Asili, J.; Tayarani-Najaran, Z. Safranal protects against betaamyloid peptide-induced cell toxicity in PC12 cells via MAPK and PI3 K pathways. Metabolic Brain Disease 2019, 34, 165-172, https://doi.org/10.1007/s11011-018-0329-9.

140. Patil, D.N.; Patil, S.A.; Sistla, S.; Jadhav, J.P. Comparative biophysical characterization: A screening tool for acetylcholinesterase inhibitors. PLoS ONE 2019, 14, https://doi.org/10.1371/journal.pone.0215291.

141. Chalatsa, I.; Arvanitis, D.A.; Koulakiotis, N.S.; Giagini, A.; Skaltsounis, A.L.; Papadopoulou-Daifoti, Z.; Tsarbopoulos, A.; Sanoudou, D. The Crocus sativus Compounds trans-Crocin 4 and trans-Crocetin Modulate the Amyloidogenic Pathway and Tau Misprocessing in Alzheimer Disease Neuronal Cell Culture Models. Front Neurosci 2019, 13, https://doi.org/10.3389/fnins.2019.00249.

142. Inoue, E.; Shimizu, Y.; Masui, R.; Hayakawa, T.; Tsubonoya, T.; Hori, S.; Sudoh, K. Effects of saffron and its constituents, crocin-1, crocin-2, and crocetin on alpha-synuclein fibrils. J Nat Med 2018, 72, 274-279, https://doi.org/10.1007/s11418-017-1150-1.

143. Tiribuzi, R.; Crispoltoni, L.; Chiurchiù, V.; Casella, A.; Montecchiani, C.; Del Pino, A.M.; Maccarrone, M.; Palmerini, C.A.; Caltagirone, C.; Kawarai, T.; Orlacchio, A.; Orlacchio, A.Trans-crocetin improves amyloid-beta degradation in monocytes from Alzheimer's Disease patients. J Neurol Sci 2017, 372, 408-412, https://doi.org/10.1016/j.jns.2016.11.004.

144. Seto, S.W.; Chang, D.; Ko, W.M.; Zhou, X.; Kiat, H.; Bensoussan, A.; Lee, S.M.; Hoi, M.P.; Steiner, G.Z.; Liu, J. Sailuotong Prevents Hydrogen Peroxide $(\mathrm{H}(2) \mathrm{O}(2))$-Induced Injury in EA.hy926 Cells. Int J Mol Sci 2017, 18, https://doi.org/10.3390/ijms18010095.

145. Amin, H.; Nieus, T.; Lonardoni, D.; Maccione, A.; Berdondini, L. High-resolution bioelectrical imaging of A $\beta$-induced network dysfunction on CMOS-MEAs for neurotoxicity and rescue studies. Scientific reports 2017, 7, https://doi.org/10.1038/s41598-017-02635-x.

146. Morelli, S.; Salerno, S.; Piscioneri, A.; Tasselli, F.; Drioli, E.; De Bartolo, L. Neuronal membrane bioreactor as a tool for testing crocin neuroprotective effect in Alzheimer's disease. Chem Eng J 2016, 305, 69-78, https://doi.org/10.1016/j.cej.2016.01.035.

147. Matsumura, S.; Murata, K.; Yoshioka, Y.; Matsuda, H. Search for $\beta$-Secretase Inhibitors from Natural Spices. Nat Prod Commun 2016, 11, 507-510.

148. Nanasombat, S.; Thonglong, J.; Jitlakha, J. Formulation and characterization of novel functional beverages with antioxidant and anti-acetylcholinesterase activities. Bioact Compd Health Dis 2015, 5, 1-16, https://doi.org/10.31989/ffhd.v5i1.162.

149. Karakani, A.M.; Riazi, G.; Mahmood Ghaffari, S.; Ahmadian, S.; Mokhtari, F.; Jalili Firuzi, M.; Zahra Bathaie, S. Inhibitory effect of corcin on aggregation of 1N/4R human tau protein in vitro. Iran J Basic Med Sci 2015, 18, 485-492.

150. Yoshino, Y.; Ishisaka, M.; Umigai, N.; Shimazawa, M.; Tsuruma, K.; Hara, H. Crocetin Prevents Amyloid 31-42-Induced Cell Death in Murine Hippocampal Cells. Pharmacology \&amp; Pharmacy 2014, 5, https://doi.org/10.4236/pp.2014.51007.

151. Ghahghaei, A.; Bathaie, S.Z.; Kheirkhah, H.; Bahraminejad, E. The protective effect of crocin on the amyloid fibril formation of a 342 peptide in vitro. Cell Mol Biol Lett 2013, 18, 328-339, https://doi.org/10.2478/s11658-013-0092-1. 
152. Ghahghaei, A.; Bathaie, S.Z.; Bahraminejad, E. Mechanisms of the Effects of Crocin on Aggregation and Deposition of A beta 1-40 Fibrils in Alzheimer's Disease. Int J Pept Res Ther 2012, 18, 347-351, https://doi.org/10.1007/s10989-012-9308-X.

153. Geromichalos, G.D.; Lamari, F.N.; Papandreou, M.A.; Trafalis, D.T.; Margarity, M.; Papageorgiou, A.; Sinakos, Z. Saffron as a source of novel acetylcholinesterase inhibitors: Molecular docking and in vitro enzymatic studies. J Agric Food Chem 2012, 60, 6131-6138, https://doi.org/10.1021/jf300589c.

154. Ahn, J.H.; Hu, Y.; Hernandez, M.; Kim, J.R. Crocetin inhibits beta-amyloid fibrillization and stabilizes betaamyloid oligomers. Biochem Biophys Res Commun 2011, 414, 79-83, https://doi.org/10.1016/j.bbrc.2011.09.025.

155. Ebrahim-Habibi, M.B.; Amininasab, M.; Ebrahim-Habibi, A.; Sabbaghian, M.; Nemat-Gorgani, M. Fibrillation of $\alpha$-lactalbumin: Effect of crocin and safranal, two natural small molecules from Crocus sativus. Biopolymers 2010, 93, 854-865, https://doi.org/10.1002/bip.21477.

156. Nam, K.N.; Park, Y.M.; Jung, H.J.; Lee, J.Y.; Min, B.D.; Park, S.U.; Jung, W.S.; Cho, K.H.; Park, J.H.; Kang, I.; Hong, J.W.; Lee, E.H. Anti-inflammatory effects of crocin and crocetin in rat brain microglial cells. Eur J Pharmacol 2010, 648, 110-116, https://doi.org/10.1016/j.ejphar.2010.09.003.

157. Gholamhoseinian, A.; Moradi, M.N.; Sharifi-far, F. Screening the methanol extracts of some Iranian plants for acetylcholinesterase inhibitory activity. Res Pharm Sci 2009, 4, 105-112.

158. Papandreou, M.A.; Kanakis, C.D.; Polissiou, M.G.; Efthimiopoulos, S.; Cordopatis, P.; Margarity, M.; Lamari, F.N. Inhibitory activity on amyloid-beta aggregation and antioxidant properties of Crocus sativus stigmas extract and its crocin constituents. J Agric Food Chem 2006, 54, 8762-8768, https://doi.org/10.1021/jf061932a.

159. Soeda, S.; Ochiai, T.; Paopong, L.; Tanaka, H.; Shoyama, Y.; Shimeno, H. Crocin suppresses tumor necrosis factor- $\alpha$-induced cell death of neuronally differentiated PC-12 cells. Life Sci 2001, 69, 2887-2898, https://doi.org/10.1016/S0024-3205(01)01357-1.

160. Abe, K.; Sugiura, M.; Shoyama, Y.; Saito, H. Crocin antagonizes ethanol inhibition of NMDA receptormediated responses in rat hippocampal neurons. Brain Res 1998, 787, 132-138, https://doi.org/10.1016/S0006-8993(97)01505-9.

161. Sugiura, M.; Shoyama, Y.; Saito, H.; Abe, K. Crocin (crocetin di-gentiobiose ester) prevents the inhibitory effect of ethanol on long-term potentiation in the dentate gyrus in vivo. J Pharmacol Exp Ther 1994, 271, 703-707.

162. Moazen-Zadeh, E.; Abbasi, S.H.; Safi-Aghdam, H.; Shahmansouri, N.; Arjmandi-Beglar, A.; Hajhosseinn Talasaz, A.; Salehiomran, A.; Forghani, S.; Akhondzadeh, S. Effects of Saffron on Cognition, Anxiety, and Depression in Patients Undergoing Coronary Artery Bypass Grafting: A Randomized Double-Blind PlaceboControlled Trial. J Altern Complement Med 2018, 24, 361-368, https://doi.org/10.1089/acm.2017.0173.

163. Akouchekian, S.; Omranifard, V.; Maracy, M.; Pedram, A.; Zefreh, A. Efficacy of herbal combination of sedge, saffron, and Astragalus honey on major neurocognitive disorder. J Res Med Sci 2018, 23, https://doi.org/10.4103/jrms.JRMS_949_17.

164. Cicero, A.F.; Bove, M.; Colletti, A.; Rizzo, M.; Fogacci, F.; Giovannini, M.; Borghi, C. Short-Term Impact of a Combined Nutraceutical on Cognitive Function, Perceived Stress and Depression in Young Elderly with Cognitive Impairment: A Pilot, Double-Blind, Randomized Clinical Trial. J Prev Alzheimers Dis 2017, 4, $12-15$.

165. Tsolaki, M.; Karathanasi, E.; Lazarou, I.; Dovas, K.; Verykouki, E.; Karakostas, A.; Georgiadis, K.; Tsolaki, A.; Adam, K.; Kompatsiaris, I.; Sinakos, Z. Efficacy and safety of crocus sativus L. in patients with mild cognitive impairment: One year single-blind randomized, with parallel groups, clinical trial. J Alzheimers Dis 2016, 54, 129-133, https://doi.org/10.3233/JAD-160304.

166. Liang, J.H.; Li, F.; Wei, C.B.; Song, H.Q.; Wu, L.Y.; Tang, Y.; Jia, J.P. Rationale and Design of a Multicenter, Phase 2 Clinical Trial to Investigate the Efficacy of Traditional Chinese Medicine SaiLuoTong in Vascular Dementia. J Stroke Cerebrovasc Dis 2014, 23, 2626-2634, https://doi.org/10.1016/j.jstrokecerebrovasdis.2014.06.005.

167. Farokhnia, M.; Sabet, M.S.; Iranpour, N.; Gougol, A.; Yekehtaz, H.; Alimardani, R.; Farsad, F.; Kamalipour, M.; Akhondzadeh, S. Comparing the efficacy and safety of Crocus sativus L. with memantine in patients with moderate to severe Alzheimer's disease: a double-blind randomized clinical trial. Hum Psychopharmacol 2014, 29, 351-359, https://doi.org/10.1002/hup.2412.

168. Akhondzadeh, S.; Shafiee Sabet, M.; Harirchian, M.H.; Togha, M.; Cheraghmakani, H.; Razeghi, S.; Hejazi, S.S.; Yousefi, M.H.; Alimardani, R.; Jamshidi, A.; Rezazadeh, S.-A.; Yousefi, A.; Zare, F.; Moradi, A.; Vossoughi, A. A A 22-week, multicenter, randomized, double-blind controlled trial of Crocus sativus in the treatment of mild-to-moderate Alzheimer's disease. Psychopharmacology 2010, 207, 637-643, https://doi.org/10.1007/s00213-009-1706-1.

169. Akhondzadeh, S.; Shafiee Sabet, M.; Harirchian, M.H.; Togha, M.; Cheraghmakani, H.; Razeghi, S.; Hejazi, S.S.; Yousefi, M.H.; Alimardani, R.; Jamshidi, A.; Rezazadeh, S.A.; Yousefi, A.; Zare, F.; Moradi, A.; Vossoughi, A. Saffron in the treatment of patients with mild to moderate Alzheimer's disease: a 16-week, randomized and placebo-controlled trial. $J$ Clin Pharm Ther 2010, 35, 581-588, https://doi.org/10.1111/j.1365-2710.2009.01133.x. 
170. Moshiri, M.; Vahabzadeh, M.; Hosseinzadeh, H. Clinical applications of saffron (Crocus sativus) and its constituents: a review. Drug Res 2015, 65, 287-295, https://doi.org/10.1055/s-0034-1375681.

171. Mohamadpour, A.; Ayati, Z.; Parizadeh, M.R.; Rajbai, O.; Hosseinzadeh, H. Safety Evaluation of Crocin (a constituent of saffron) Tablets in Healthy Volunteers. Iran J Basic Med Sci 2013, 16, 39-46.

172. Hosseini, A.; Razavi, B.M.; Hosseinzadeh, H. Pharmacokinetic properties of saffron and its active components. Eur J Drug Metab Pharmacokinet 2018, 43, 383-390, https://doi.org/10.1007/s13318-0170449-3.

173. Moratalla-López, N.; Bagur, M.J.; Lorenzo, C.; Martínez-Navarro, M.; Salinas, M.R.; Alonso, G.L. Bioactivity and bioavailability of the major metabolites of Crocus sativus L. Flower. Molecules 2019, 24, 2827, https://doi.org/10.3390/molecules24152827.

174. Rahaiee, S.; Shojaosadati, S.A.; Hashemi, M.; Moini, S.; Razavi, S.H. Improvement of crocin stability by biodegradeble nanoparticles of chitosan-alginate. Int $J$ Biol Macromol 2015, 79, 423-432, https://doi.org/10.1016/j.ijbiomac.2015.04.041. 